Sharif University of Technology
Scientia Iranica
SCIENTIA $\quad \begin{gathered}\text { Transactions D: Computer Science \& Engineering and Electrical Engineering } \\ \text { w }\end{gathered}$

\title{
Repeating average filter for noisy texture classification
}

\author{
M.H. Shakoor* and F. Tajeripour \\ School of Electrical and Computer Engineering, Shiraz University, Shiraz, P.O. Box 71348-51154, Iran. \\ Received 8 November 2015; received in revised form 3 March 2016; accepted 28 June 2016
}

\author{
KEYWORDS \\ Local binary pattern; \\ Texture classification; \\ Repeating average \\ filter; \\ Completed local \\ binary pattern; \\ Noise robustness.
}

\begin{abstract}
In this paper, it is shown that repeating average filter increases the uniform patterns of noisy textures and, consequently, increases the classification accuracy of textures. In other words, for noisy textures, first, an average filter, such as $3 \times 3$ mean filter, is applied to each image; then, a feature extraction method, such as LBP, is used to extract features of the filtered image. The more value of noise, the more repeating of average filter should be applied to textures. Moreover, it is shown that by repeating the $3 \times 3$ average filter for textures, the variance of texture decreases, then increases. Thus, average filter must be repeated while the variance of image decreases and when the variance starts increasing, it must be stopped. Using convolution to apply average filter for an image takes so much time; therefore, a simple technique is proposed in this paper that increases the speed of average filtering significantly. After noise reduction, by using LBP operator, features of texture are extracted for classification. Implementations on Outex, CUReT, and UIUC datasets determine that the performance of the proposed method is better than that of some advanced noise-resistant LBP variants such as BRINT and CRLBP.
\end{abstract}

(C) 2017 Sharif University of Technology. All rights reserved.

\section{Introduction}

Texture analysis plays important roles in image processing and computer vision. There are many applications that use texture classification and segmentation. Some applications such as fabric defect detection $[1,2]$, medical image analyzing [3], remote sensing [4], face detection [5], and image retrieval [6] are related to texture analysis. The main point of texture analyzing and classification is feature extraction. In the last decades, many types of texture features extraction have been proposed. One of the first and important types of texture features extraction is statistical, including some methods such as co-occurrence matrix [7] and local binary patterns [8]. The second type includes model-based methods such as hidden Markova [9], autocorrelation [10], and autoregressive [11] models.

*. Corresponding author. Tel.: +98 7138203540 E-mail address: mhshakoor@shirazu.ac.ir, (M. H. Shakoor); tajeri@shirazu.ac.ir (F. Tajeripour)
Anisotropic Circular Gaussian MRF (ACGMRF) [12] is an improved version of the Gaussian Markov random field method [13]. It is rotation-invariant and sensitive to directional features. The third group of these methods is related to structural methods such as topological texture descriptors [14] and morphological methods [15]. Finally, the fourth analyzing methods are frequency-based or filter-based, such as some Gabor and wavelet methods. These methods capture visual properties such as spatial localization, spatial frequency, and orientation of the structures present in the image. Widely employed for object recognition, Gabor filters present illumination invariance since they detect invariant spatial frequency [16]. There are some Gabor techniques such as Traditional Gabor Filters (TGF) [17], Circular Gabor Filters (CGF) [18], and Normal Gabor Filters (NGF) [19]. Some waveletbased algorithms [20-22], such as Daubechies wavelet transform features (DBWP) [23], are related to the frequency-based method.

One of the statistical methods of texture descrip- 
tors is Gray Level Co-occurrence Matrices (GLCM) [24]. Besides the original version of the GLCM, several variations have been proposed. Focusing on optimization, Clausi and Jernigan [25] used linked lists exploiting the scarcity of the co-occurrence matrices to reduce the computation time. Some extensions of the GLCM have been proposed. For increasing the discriminability of the descriptors, Gelzinis et al. [26] extracted descriptors considering simultaneously different values for parameter $d$. Walker et al. [27] proposed co-occurrence matrix-based features by weighted summation of GLCM elements from areas presenting high discrimination. Furthermore, addition of color information has been considered for cooccurrence matrices [28]. Multi-scale analysis has also been performed using the GLCM. Hu [29] and Pacifici et al. [30] consider multiple scales by changing the window size from which the GLCM descriptors are extracted. Rakwatin et al. [31] proposed that the image be rescaled to different sizes, extracting cooccurrence descriptors from each size. Nguyen-Duc et al. [32] obtained improved results for content-based image retrieval employing a combination of contourlet transform [33] and GLCM. First, the contourlet transform was performed for four sub-bands of the image; then, the GLCM features were extracted from each one.

The development and analysis of low-level feature descriptors have been widely considered in the past years. Among the vastly employed methods are the Scale-Invariant Feature Transform (SIFT) [34], Speeded Up Robust Feature (SURF) [35], Histogram of Oriented Gradients (HOG) [36], and Gradient Location and Orientation Histogram (GLOH) [37].

One of the most popular and simple methods for texture features extraction is Local Binary Pattern (LBP). This method is a statistical one that extracts uniform properties of each texture. For the first time, Ojala et al. proposed LBP [38]. It is an operator to describe local patterns and it has achieved high performance for classification results on many kinds of texture datasets [39]. LBP is a method that is not sensitive to monotonic gray scale change.

The first goal of LBP is related to texture classification, however it has been used for some applications such as face recognition [40], dynamic texture recognition [41], and shape localization [42]. Before introducing LBP, a similar method, i.e. Census Transform (CT), was proposed by Zabih et al. [43]. The first version of LBP provided too many features and was sensitive to rotation. Therefore, Ojala [44] offered two rotation-invariant LBP methods. $\operatorname{LBP}_{(P, R)}^{r i}$ is a rotation-invariant type of LBP; however, it also extracts too many features from each texture. Furthermore, Ojala proposed $\mathrm{LBP}_{P, R}^{u 2}$ that was not rotationinvariant and prepared too many features, but it was robust to noise. One of the most important methods proposed by Ojala et al. was $\operatorname{LBP}_{(P, R)}^{r i u 2}$ [45]. This method not only extracted smaller numbers and high discriminative features, but also was rotation-invariant and became the most popular since it decreased number of the features significantly and obtained high discriminative ability.

There are some drawbacks in LBP. It is a local operator, so it is sensitive to noise. Therefore, some noise-robust LBP methods are introduced. Jin et al. introduced Improved Local Binary Pattern (ILBP) [46]. It is similar to simple LBP, but in ILBP the mean value of the neighborhood and center points is used instead of the center point. One of the most important and simple noise-resistant LBP methods is LTP. Tan and Triggs [47] proposed Local Ternary Pattern (LTP) to quantize the difference between a pixel and its neighbors into three levels. Dominant LBP (DLBP) [19] was introduced by Liao et al. It used the most frequently occurred patterns to capture descriptive textural features. It selected $80 \%$ of the most frequently appeared patterns from histogram of LBP and the other $20 \%$ of patterns that contained almost non-uniform noise were removed from features. Another noise-robust LBP is fuzzy local binary pattern FLBP [48] or soft LBP [49]. In this method, each pixel position may contribute to several bins in the histogram of possible patterns by different membership $p$ values. The FLBP is a very time consuming method. Hafiane et al. proposed Median Binary Patterns (MBP) [50]. MBP used median gray value of the neighborhood points instead of the center point. Fathi et al. [51] proposed a noise-tolerant method (NTLBP) that used circular majority voting. This method regrouped the non-uniform LBP patterns to obtain better performance. Ren et al. [52] proposed an efficient NoiseResistant Local Binary Pattern (NRLBP) approach. The NRLBP method restored some local structures of the image that were cropped by noise; however, it was very time consuming and could not be generalized to neighborhoods with larger scales. Therefore, it was efficient only for small neighborhoods such as $R=1$ and $P=8$. Lui et al. [53] proposed Binary Rotation Invariant and Noise Tolerant (BRINT) texture classification method that used mean of the neighbor points for LBP. This method decreased the effects of noise by using the mean value of some sequential neighbor points on the circular patch. In other words, it reduced the noise value of the neighbor points. BRINT used average of angular points (points on the circle of neighborhood) instead of neighbor points of LBP. Some methods, such as Completed Robust Local Binary Pattern (CRLBP) [54], used average filter and Weighted Local Gray level (WLG) to reduce the noise. In CRLBP, the value of each center point in a $3 * 3$ local patch is replaced by its average local grey value. CRLBP used a weight value for the center point when 
it calculated the mean of the points. These methods are some of the most popular LBPs that are resistant to noise. Kylberg et al. reviewed and compared most of them in [55].

There are many applications for texture classification. Zhang and $\mathrm{Wu}$ proposed a method for fruits classification [56]. They proposed a hybrid classification method based on Fitness-Scaled Chaotic Artificial Bee Colony (FSCABC) algorithm and feed Forward Neural Network (FNN). Classification of fruits is a difficult challenge due to the numerous types of fruits. In this method, the color histogram, texture, and shape features of each fruit image are extracted to compose a feature space. Zhang and Wu showed that the combination of color histogram, Unser's texture, and shape features is more effective than any single kind of feature in classification of fruits. Zhang and $\mathrm{Wu}$ used Unser method [57] to describe the texture features for classification. Unser described a new approach [57] to the characterization of texture properties at multiple scales using the wavelet transform. The analysis used an over-complete wavelet decomposition, which yielded a description that was translation-invariant. Unser proved that the sum and difference of two random variables with the same variances are de-correlated and the principal axes of their associated joint probability function are defined. The use of the wavelet transform in texture classification processes can contribute to improving the results, but it seems to be dependent on the area and the texture types.

As it is mentioned before, CRLBP uses the average filter during LBP operations. In this paper, some average filters are used as preprocessing operation for noise reduction. Moreover, the main motivation of this paper is to repeat average filtering for more noise reduction. Average filter is used more than one time to increase the percentage of uniform patterns and decrease the noise effects. Implementation shows that repeating average filter for low SNR textures increases the classification accuracy significantly and provides performance that is better than that of some advanced and state-of-the-art noise-robust LBP variants. Repeating average filter by convolving it with noisy image takes so much time; therefore, in this paper, a simple technique is proposed that increases the speed of average filtering noticeably and increases the speed of preprocessing around 30 times.

This paper is organized as follows: In Section 2, LBP and some of the last versions of LBP are explained. Section 3 presents the proposed methods. Experimental results and conclusion are reported in Sections 4 and 5 , respectively.

\section{Brief review of some LBPs}

In this section, a brief review of Local Binary Pat- tern (LBP), CLBP, and CRLBP is prepared. These methods are related to the implementation part of the proposed method.

\subsection{Local Binary Pattern (LBP)}

LBP provides binary codes by comparing $P$ points of the neighboring pixels with respect to the center point. It generates a binary code 0 if the value of the neighboring pixel is smaller than the center value of patch. Otherwise, it generates a binary code 1 . Then, the binary codes are multiplied by the corresponding weights and the results are outlined to generate an LBP code. This value is calculated as follows:

$$
\operatorname{LBP}_{P, R(x, y)}=\sum_{i=0}^{P-1} s\left(g_{i}-g_{c}\right) 2^{i},
$$

where $g_{c}$ is the pixel value of the center point and $g_{i}$ is the pixel value of the $i$ th neighboring pixel, $P$, is the number of neighboring pixels, and $R$ is the radius.

$$
s\left(g_{i}-g_{c}\right)= \begin{cases}1 & g_{i} \geq g_{c} \\ 0 & g_{i}<g_{c}\end{cases}
$$

If the square neighborhood is used, LBP provides features that are not rotation-invariant. The circular neighborhood must be used for rotation-invariant methods. To obtain rotation invariance, the original LBP is extended to a circular symmetric neighbor set of $P$ members on a circular region with radius $R$ using uniform patterns [8]. For circular neighboring points, it is necessary to interpolate some neighbor points. The rotation-invariant uniform LBP ( $\mathrm{LBP}^{\text {riu2 }}$ ) [45] can be obtained by Eqs. (3)-(5). In these equations, riu2 reflects the rotation-invariant uniform patterns that have a $U$ value of at most $2 . U$ is used to estimate the uniformity that corresponds to the number of spatial transitions, i.e. bitwise 0/1 changes between successive bits in the circle. Furthermore, $\mathrm{LBP}^{r i}$ and $\mathrm{LBP}^{u 2}$ [44] are two other types of LBP that are used for texture classification. $\mathrm{LBP}^{r i}$ is a rotation-invariant method but $\mathrm{LBP}^{u 2}$ is not. Because of the high number of features, both of these methods are very time consuming and are not used for real-time and fast texture processing:

$$
\begin{aligned}
& \operatorname{LBP}_{P, R}^{r i u 2}(x, y)= \begin{cases}\sum_{i=0}^{P-1} s\left(g_{i}-g_{c}\right) & \text { if } U\left(\operatorname{LBP}_{P, R}\right) \leq 2 \\
P+1 & \text { otherwise }\end{cases} \\
& s\left(g_{i}-g_{c}\right)= \begin{cases}1 & g_{i} \geq g_{c} \\
0 & g_{i}<g_{c}\end{cases} \\
& U\left(\operatorname{LBP}_{P, R}\right)=\left|s\left(g_{P-1}-g_{c}\right)-s\left(g_{0}-g_{c}\right)\right| \\
& +\sum_{i=1}^{P}\left|s\left(g_{i}-g_{c}\right)-s\left(g_{i-1}-g_{c}\right)\right|
\end{aligned}
$$




\subsection{Completed Local Binary Pattern (CLBP), Completed Robust Local Binary Count (CRLBP)}

Gue et al. proposed CLBP [58] method. CLBP combines the sign and magnitude codes of LBP. In CLBP, the local difference is divided into the sign (S) and magnitude $(M)$ so that CLBP_S and CLBP $M$ are made. Also, the center point of each patch is compared with the average of the entire image and CLBP_C is made. Both CLBP_S and CLBP_M produce binary strings so that they can be combined for texture classification. The most accurate result is provided by CLBP_S/M/C. In most papers [53,54] and in this paper, CLBP refers to CLBP_S/M/C. A noise-resistant CRLBP method is same as the CLBP, but it uses average value of $3 \times 3$ patch instead of each point. CRLBP uses $\alpha=1$ and 8 as weights of center point when it calculates the average value of each patch. In this paper, some average filters that use circular and square neighborhoods are used. These filters are applied to noisy textures before feature extraction.

\section{Average filtering}

In this paper, some types of average filters are used for noise reduction. Figure 1 illustrates the performance when using four types of average filter for Outex (TC10) dataset. In this figure, the classification accuracy of noisy textures for SNR $=3,5,10$, and 20 is shown. Also, the results of some filters are shown. Square mean filter $(s$-mean) uses a square $3 \times 3$ mask for filtering. $S$-median uses a similar mask, but it calculates the median of 9 points. $C$-mean is a mean filter that calculates the mean of points on a circular neighborhood $(R=1)$. It calculates the mean of 9 points that include 8 points on the circle and the center point. It uses the weights $\alpha=1$ and 8 for the center point. The results are determined for LBP_S, LBP_M, LTP, and CLBP. The best accuracy is obtained for CLBP. Therefore, in his paper, the results of repeating of the average filter for CLBP are used and they are named Repeat Filter CLBP or RF_CLBP.

The plots in Figure 1 indicate that some filters, such as $c$-mean $(\alpha=1)$ and $s$-mean, perform better than $c$-mean $(\alpha=8)$ and $S$-median. The performance of $c$-mean $(\alpha=1)$ is similar to that of $s$-mean. However, for some cases, the performance of $c$-mean $(\alpha=1)$ is slightly better than that of $s$-mean. $C$-mean requires interpolation step to calculate some points on the circle. Thus, in this paper, $s$-mean filter is used.

\subsection{Repeating average filter}

In some methods such as CRLBP [54] mean filter of $3 \times 3$ is applied one time to all points of noisy texture to decrease the noise. In this paper, it is shown that if average filter is applied more than one time to a noisy texture, better accuracy of classification can be obtained. In other words, the more value of noise the more number of average filters should be applied to noisy texture. Some feature extraction methods such as LBP use uniform patterns to extract discriminative features of textures. The proposed algorithm is shown
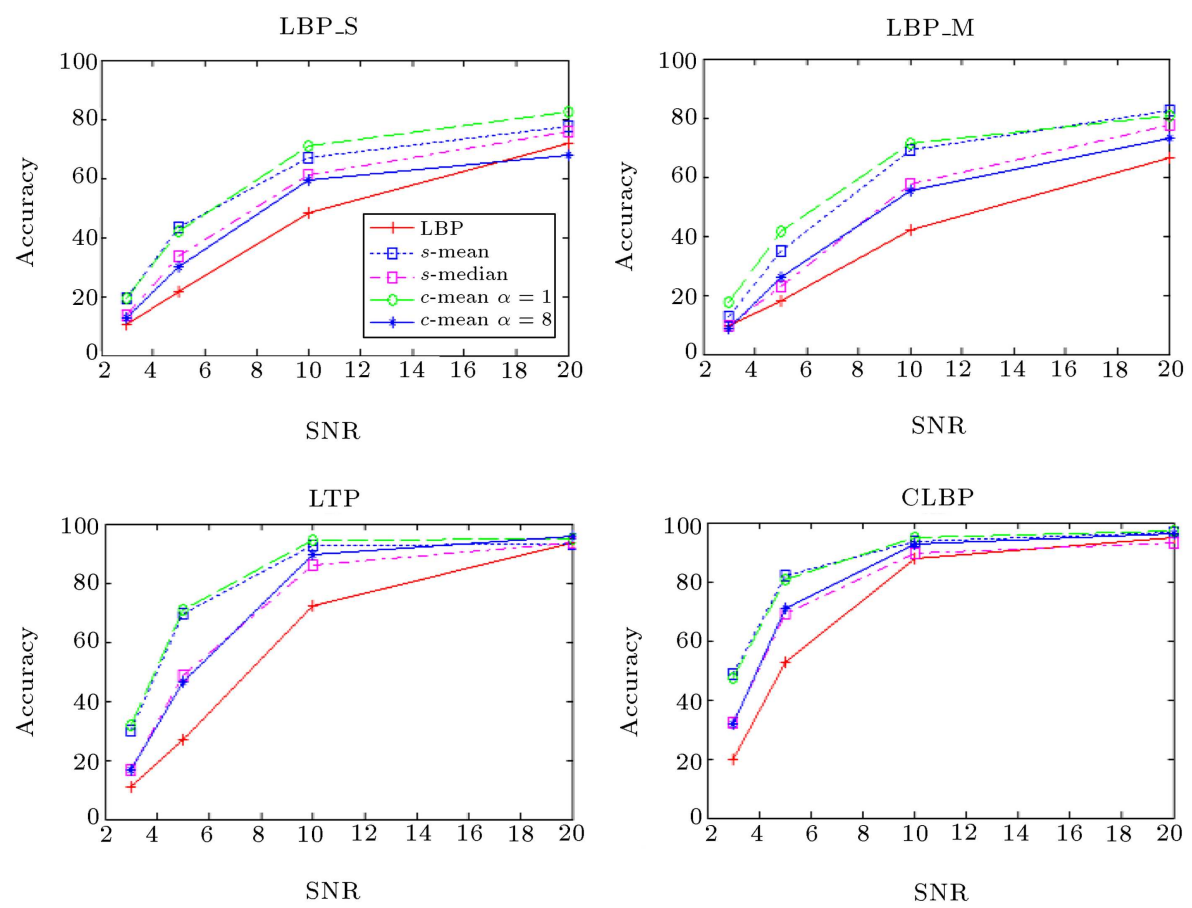

Figure 1. Comparison of the performances of some average filters for noisy Outex (TC10) textures with four variants of LBP $(R=1$ and $P=8)$ and different SNR values. 


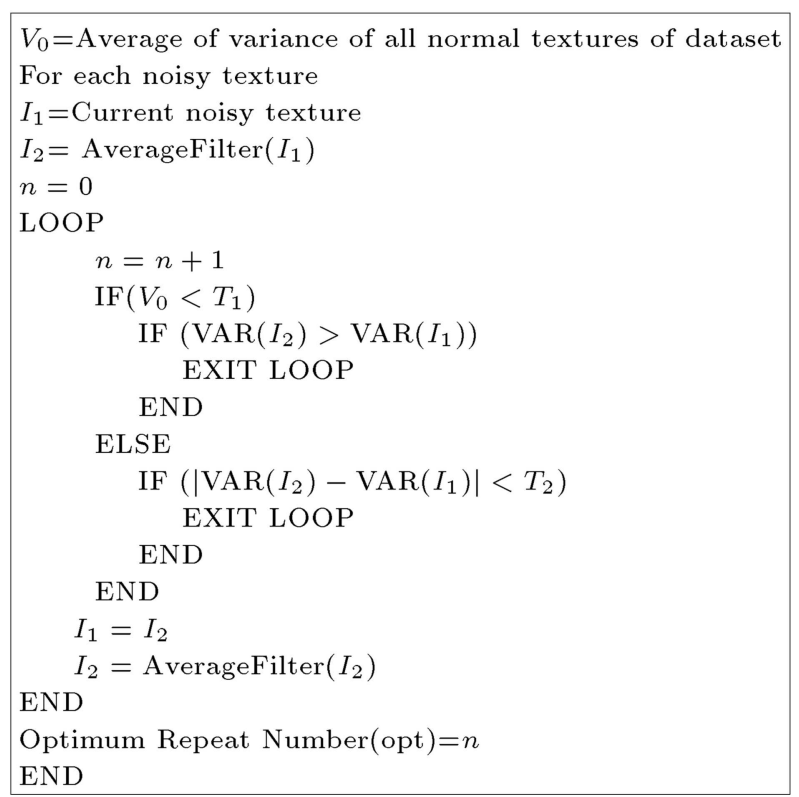

Figure 2. Pseudo code of estimation of optimum number for repeating the average filter.

in Figure 2. When a texture is corrupted by noise, the percentage of uniform patterns decreases significantly. However, by using average filter, it is possible to increase this percentage. If average filter is applied to a noisy texture, the percentage of uniform patterns increases. Therefore, by repeating this filtering operation, it is possible to extract more efficient features by LBP operator.

The implementation shows that for low SNR or very noisy textures, the number of repeats of average filter must be high, such as 5 or 6 , and for low noise or high SNR, it must be used only one or two. If the number of repeats of using average filter is small, it may not reduce the noise efficiently. On the other hand, if this number is too large, it corrupts the texture edges and leads to worse results. Therefore, it is necessary to find the best number of repetitions. Moreover, it is necessary to use small mask for average filter to save the local edge and contrast of textures. Therefore, in this paper, a $3 \times 3$ average mask is used.

Figures 3 and 4 determine the percentage of uniform patterns of LBP versus the number of repeats of the average mask for Outex and CUReT datasets, respectively. In both of these figures, $R=1$ and $P=8$. In Figure 3, the percentage of uniform patterns is around $90 \%$ without noise. In this figure, SNR = 5. It shows that the percentage of uniform patterns of noisy textures is around $75 \%$ and this percentage reaches $88 \%$ after the first time of applying average filter. After the second use of average filter, it reaches $95 \%$ and after that, it increases slightly. In addition, Figure 4 shows the similar trend for texture of CUReT.

Figures 3 and 4 indicate the uniform percentage

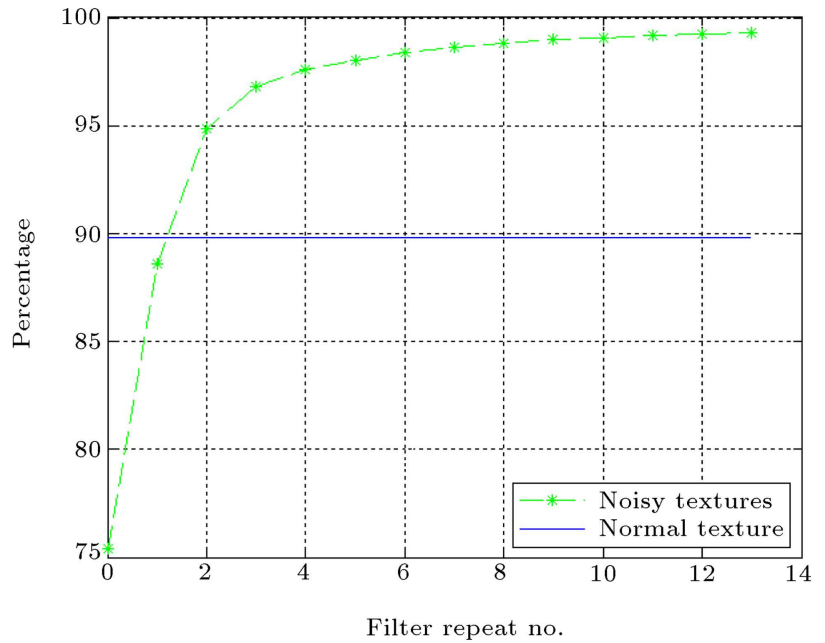

Figure 3. Uniform percentage of LBP_S versus number of repeats of average filter for noisy Outex textures $(\mathrm{SNR}=5)$.

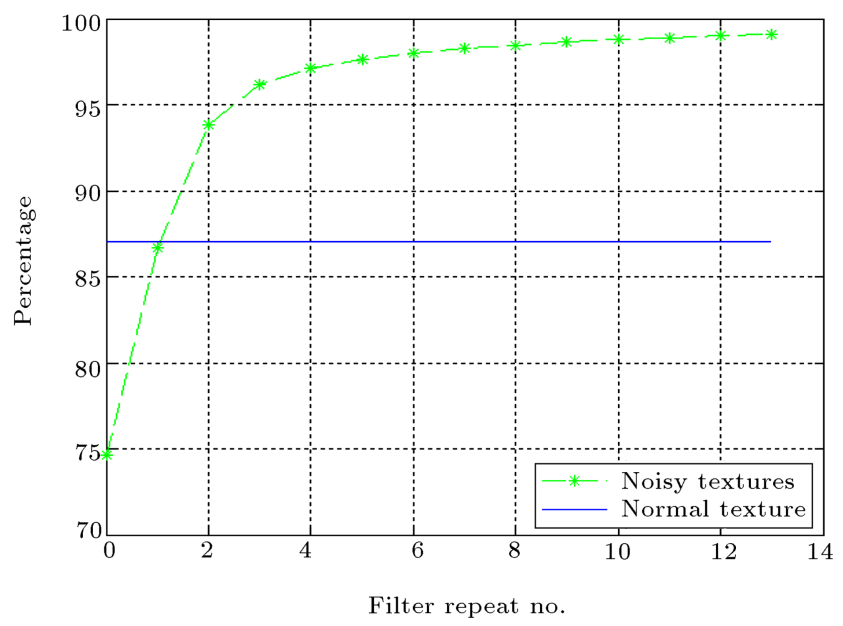

Figure 4. Uniform percentage of LBP_S versus number of repeats of average filter for noisy CUReT textures $(\mathrm{SNR}=3)$.

for a value of SNR. Figure 5 determines the uniform percentage for Outex dataset for different values of SNR. In this figure, the uniform percentage for noisy, normal, and filtered textures is shown. The filtered textures are filtered by using average filter for 3,7 , and 10 times. According to this figure, the more number of repeats of average filters, the more percentage of uniform patterns can be obtained.

\subsection{Optimum number of repeats for average filter}

One of the important points is the optimal number of repeats for the average mask. In addition, average mask decreases the noise in a noisy texture and increases the uniform patterns of texture; also, it corrupts some edge and local texture information. Therefore, it is necessary to obtain the optimum or best number for repeating of the average mask. According to Figures 3 


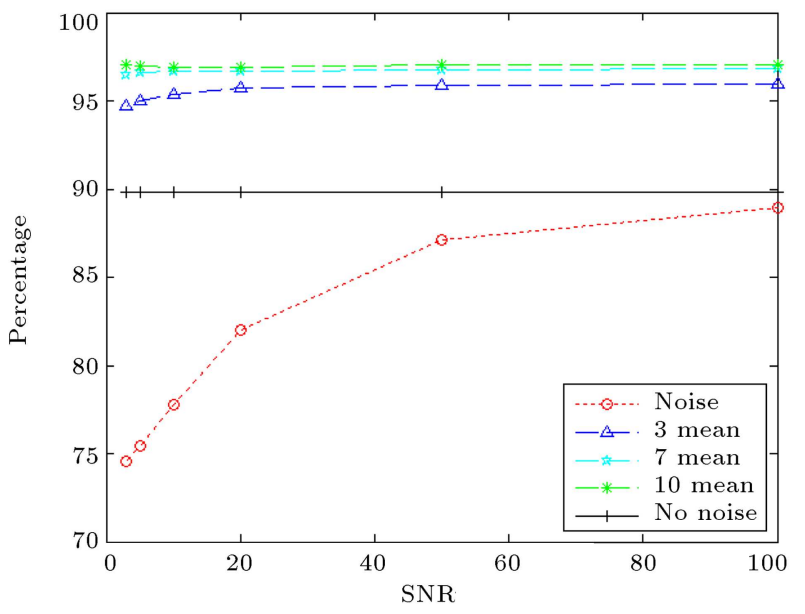

Figure 5. Uniform percentage of Outex for different values of SNR.

and 4 , the percentage of uniform patterns increases significantly at the first, second, and third times of using average mask, but after that it reaches a plateau. Table 1 indicates the summary of implementation for repeating of average mask. It shows the optimum numbers of repeats of average mask for Outex (TC10 and TC13), CUReT, and UIUC datasets. In other words, in this table, the number of repeats to reach the highest accuracy (Max. Acc.) is shown for each SNR value and dataset. In addition, the number of repeats to record the lowest variance of image is determined in Min. Var. columns. It is important to note that the numbers in Table 1 may change for a different run of implementation. It is because of random behavior of noise. However, the changes are not large and they may be 1 or 2 .

Noise increases the variance of image. On the other hand, using average filter reduces variance. Repeating average filter decreases the variance of image. In this paper, it is shown that for texture images by repeating average filter the variance decreases, then it increases. Therefore, the average filtering operation should continue until the average variance of each image reaches the minimum value. In other words, the optimum value for repeating average filter is obtained

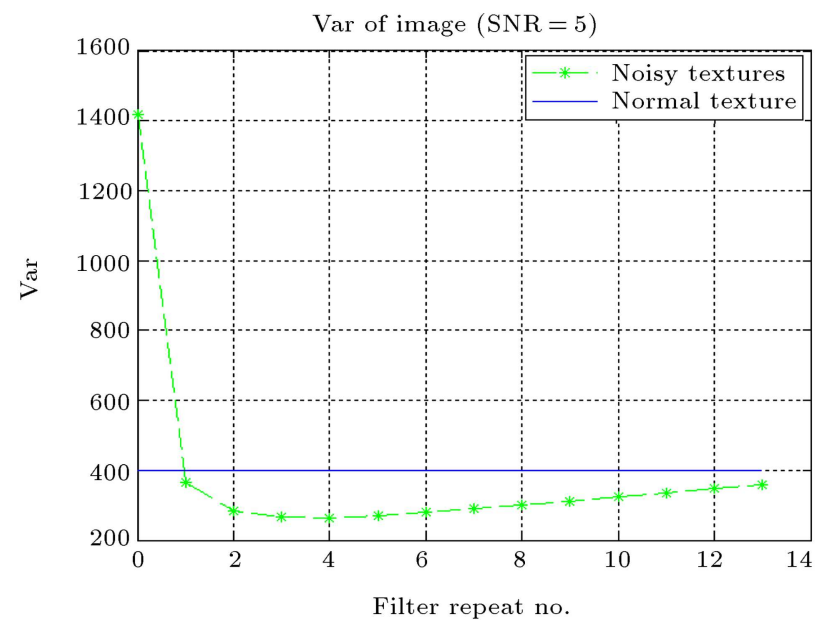

Figure 6. Variance of filtered noisy image of CUReT dataset versus number of the applications of the filter for $\mathrm{SNR}=5$.

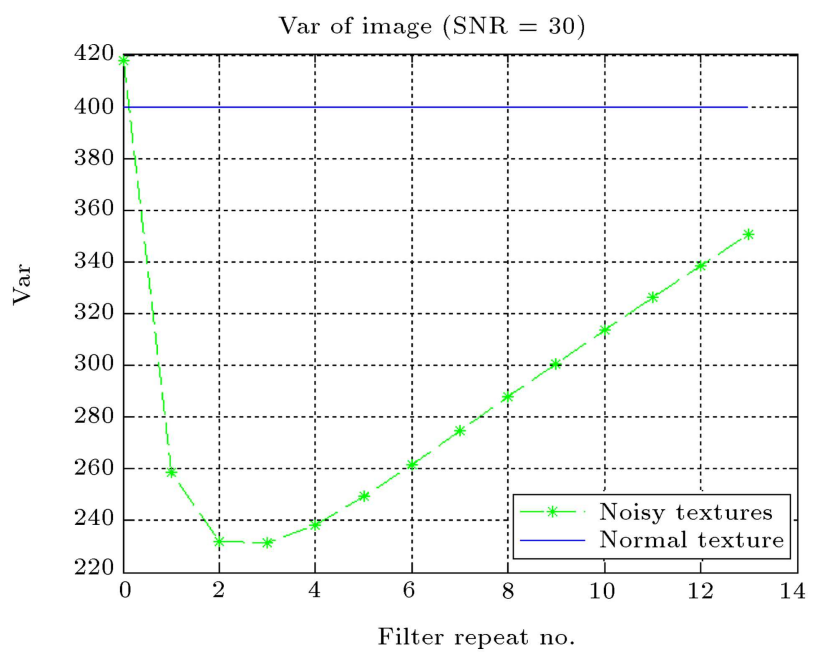

Figure 7. Variance of filtered noisy image of CUReT dataset versus number of the applications of the filter for $\mathrm{SNR}=30$.

when variance reaches the lowest values. Figures 6 to 9 show the variance (mean variance of all textures) of filtered textures after applying the average filter for 0 to 13 times. These figures indicate that variance of noisy textures decreases, then increases by using

Table 1. The relation between max accuracy and min variance of noisy textures and number of repeats of average filter for different SNR values for Outex, CUReT, and UIUC datasets.

\begin{tabular}{|c|c|c|c|c|c|c|c|c|}
\hline \multirow[b]{2}{*}{ SNR } & \multicolumn{2}{|c|}{ Outex (TC10) } & \multicolumn{2}{|c|}{ Outex (TC13) } & \multicolumn{2}{|c|}{ CUReT } & \multicolumn{2}{|c|}{ UIUC } \\
\hline & $\begin{array}{l}\text { Max } \\
\text { Acc }\end{array}$ & $\begin{array}{l}\text { Min } \\
\text { Var }\end{array}$ & $\begin{array}{l}\text { Max } \\
\text { Acc }\end{array}$ & $\begin{array}{l}\text { Min } \\
\text { Var }\end{array}$ & $\begin{array}{l}\text { Max } \\
\text { Acc. }\end{array}$ & $\begin{array}{l}\text { Min } \\
\text { Var }\end{array}$ & $\begin{array}{c}\text { Max } \\
\text { Acc }\end{array}$ & $\begin{array}{l}\text { Min } \\
\text { Var }\end{array}$ \\
\hline 2 & 20 & 5 & 6 & 6 & 14 & 6 & 21 & 90 \\
\hline 3 & 6 & 5 & 5 & 5 & 7 & 5 & 15 & 105 \\
\hline 5 & 4 & 3 & 3 & 3 & 5 & 4 & 5 & 115 \\
\hline 10 & 3 & 2 & 4 & 3 & 2 & 3 & 4 & 110 \\
\hline 30 & 3 & 2 & 2 & 2 & 2 & 2 & 4 & 110 \\
\hline
\end{tabular}




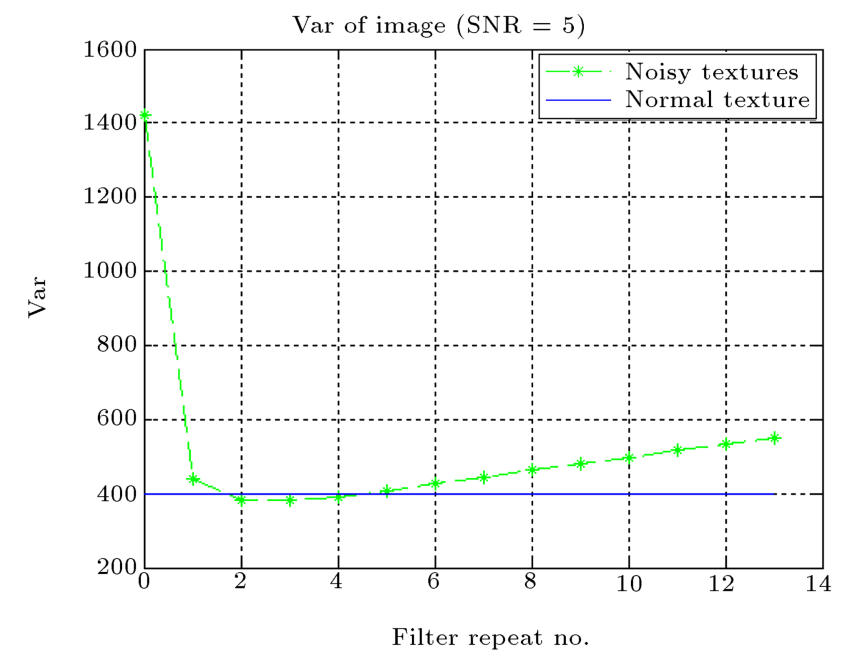

Figure 8. Variance of filtered noisy image of Outex (TC10) dataset versus number of the applications of the filter for SNR $=5$.

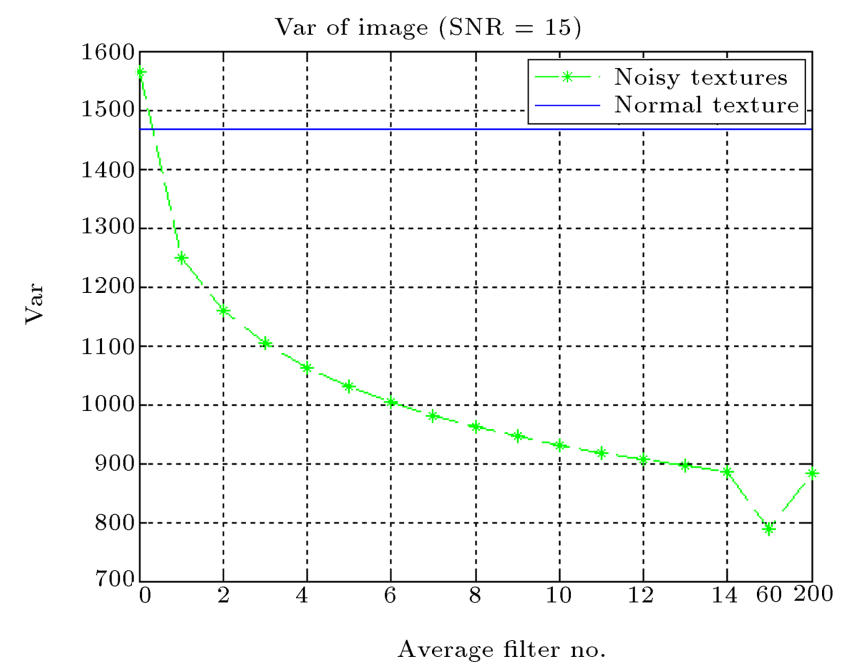

Figure 9. Variance of filtered noisy image of UIUC dataset versus number of the applications of the filter for $\mathrm{SNR}=15$.

average mask. Figure 6 shows the variance of all noisy textures of CUReT (SNR $=5$ ) versus the number of repeats of average filter. It also determines the variance of normal textures (no noise). According to this figure, the minimum value of variance is obtained when the number of repeats of the filter is four (or may be three or five). In other words, the best accuracy is obtained when the average filter is applied 4 times to noisy textures. Another example is shown in Figure 7. This figure is same as the previous figure, but SNR is 30 . Therefore, the number of repeats should be lower than that in the previous example. Figure 7 indicates that the repeat number 2 or 3 is the best because it provides minimum variance. Also, Figure 8 indicates the same trend for Outex dataset. For some datasets such as Outex and CUReT, the variance decreases by repeating average filter and after a small number of repeats, it increases. However, for some datasets such as UIUC, it decreases at first and increases after applying the average filter for more than 200 times. It is indicated in Figure 8. One of the differences of UIUC and other datasets relates to high variance of UIUC textures. The mean variance of UIUC dataset without noise is around 1480, while it is around 400 for Outex and CUReT. In other words, for some textures such as UIUC that have high variance (without noise), the highest accuracy is not reachable when variance is minimum. For these types of textures, the optimum number for repeating the average filter is obtained when the change of variance is lower than a threshold.

Table 1 determines that if the minimum variance is obtained after applying the average filter for $k$ times, the highest accuracy is achieved when the number of repeats is around $k$. Only for UIUC dataset, this relation is not true. As it is shown in Figure 9, after a large number of repeats, variance increases. However, the maximum accuracy is obtained for lower numbers of repeats. Figures 10 to 12 determine the relation between change of variance and accuracy of RF_CLBP (repeating filter CLBP) for Outex (TC10 and TC13) and CUReT datasets for some SNR values. These figures indicate that the accuracy of classification reaches a peak when variance reaches the lowest value. In Figure 10, the max accuracy for TC10 is obtained when $n=6$ and variance reaches the lowest value when $n=5$. In Figures 10 and 11, the highest accuracy is achieved when $n$ is 4 and 5 . In these figures, the minimum value of variance is achieved when $n$ is 4 and 7 , respectively. In these figures, the min value of variance is recorded when $n$ is 3 and 5 , respectively. However, as it is shown in Figure 13, the
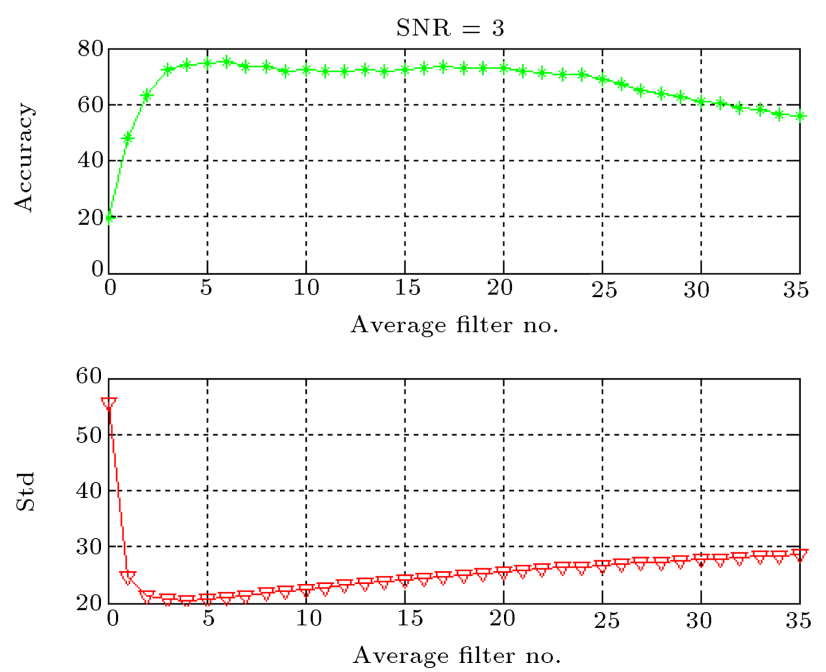

Figure 10. Standard deviation of image and accuracy of RF_CLBP of the filtered noisy image of Outex (TC10) dataset versus number of the applications of the filter for $\mathrm{SNR}=3$. 

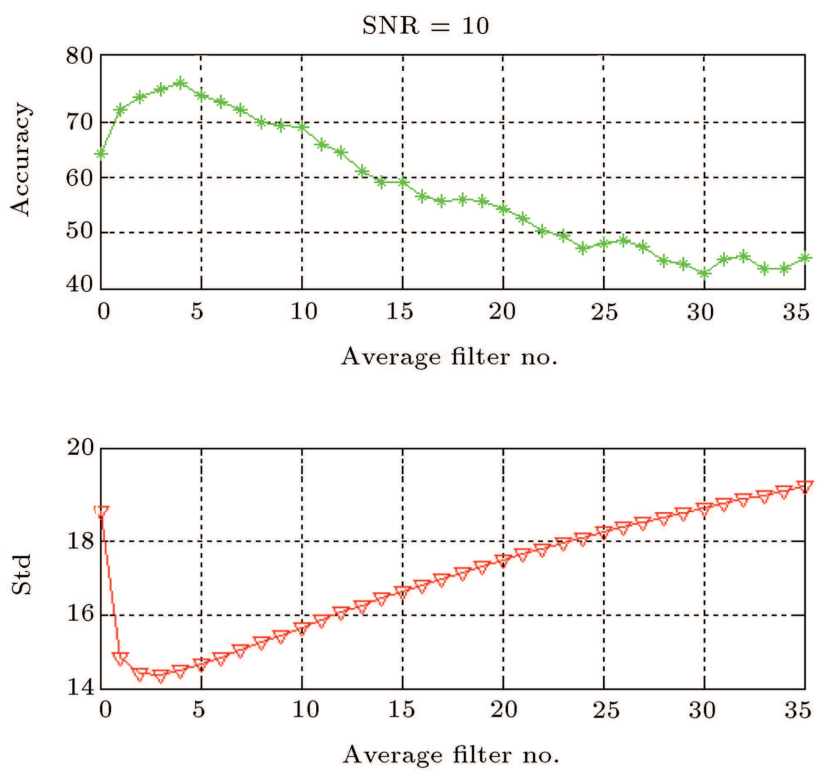

Figure 11. Standard deviation of image and accuracy of RF_CLBP of the filtered noisy image of Outex (TC13) dataset versus number of the applications of the filter for $\mathrm{SNR}=10$.
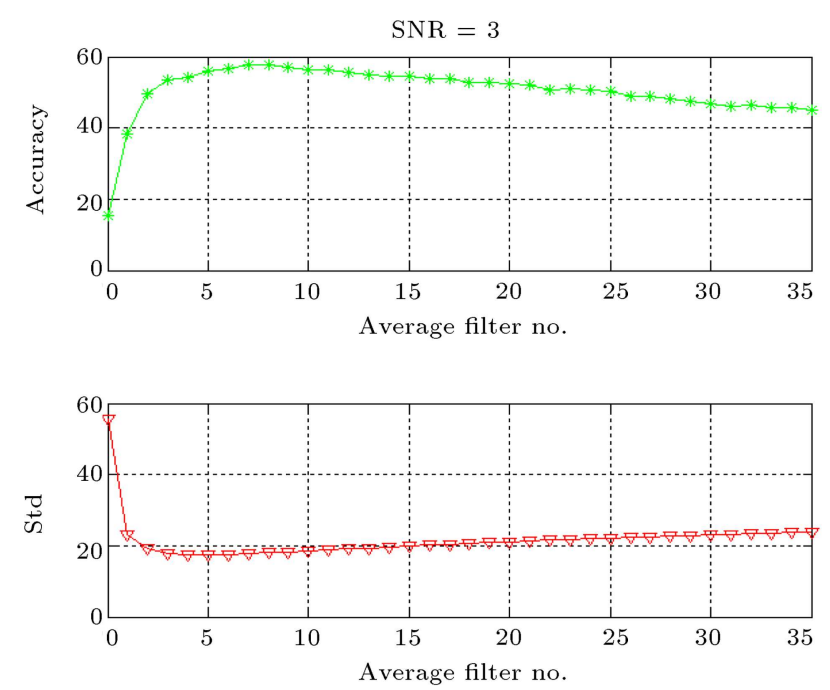

Figure 12. Standard deviation of image and accuracy of RF_CLBP of the filtered noisy image of CUReT dataset versus number of the applications of the filter for $\mathrm{SNR}=3$.

trend of change of variance and accuracy for UIUC textures does not follow this relation; UIUC is a dataset that includes high-variance images. For this type of datasets, the optimum number of repeats for the average mask is determined when the change of variance is negligible.

The pseudo code in Figure 2 estimates the optimal number of repeats of the average filter for noisy textures to reach the best accuracy. As it is mentioned in this code, for some textures such as Outex and CUReT of which the average variances are lower than $T_{1}\left(T_{1}\right.$ is around 1000), the average filter should be repeated
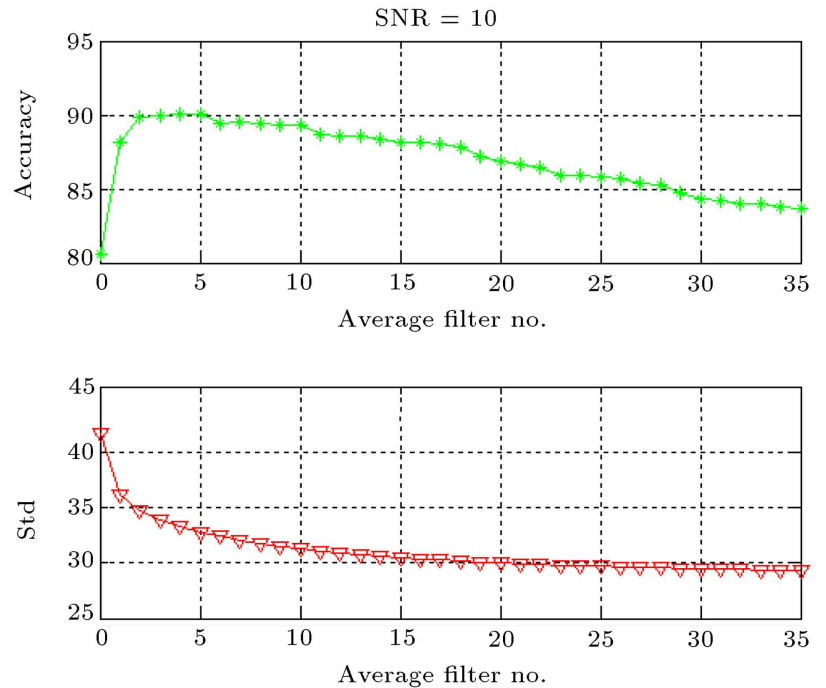

Figure 13. Standard deviation of image and accuracy of RF_CLBP of the filtered noisy image of UIUC dataset versus number of the applications of the filter for $\mathrm{SNR}=10$.

until the variance of image reaches the lowest value. As illustrated in Figures 6, 7, and 8, the average variance of these textures (without noise) is around $400\left(<T_{1}\right)$. For some textures such as UIUC, this value is around $1480\left(>T_{1}\right)$. It is shown in Figure 9. Therefore, the optimum number of repeats for average filtering is determined when the change of variance is lower than $T 2$. The value of this threshold depends on $\mathrm{V} 0 ; \mathrm{V0}$ is average variance of all normal textures (without noise).

\subsection{Fast average filtering method}

In this section, a simple technique is introduced to increase the average filtering. The circular mean filter requires interpolating neighbor points on the circular patch. For the $3 \times 3$ area, 4 point values should be interpolated from neighbor points. Therefore, to increase the speed of filtering, square mean filter is used instead of circular mean filter [59] to remove the time of interpolation. It does not have negative effects on the performance, because, as it is shown in Figure 1, the performance of circular mean filter is only slightly better than that of square mean filter.

Here, a simple technique is proposed that increases the speed of applying of square filter to an image. If image size is $M * N$, a $3 \times 3$ mask should be convolved $(M-1) *(N-1)$ times with the image, which takes too much time. To decrease this time, the noisy image $I 0$ should be shifted one pixel to eight directions, by which eight shifted images of $I 1$ to $I 8$ are provided. Then, as in Eq. (6), by calculating the average of all of the 9 images, the filtered image is provided. $I_{\text {avg }}$ is same as the noisy image that is filtered by $3 \times 3$ square mean filter. This method increases the speed of average filtering. The increase in speed depends on the size of each texture. For datasets that are used in 
this paper, using this technique increases the speed of average filtering around 30 times:

$$
I_{\mathrm{avg}}=\frac{1}{9} \sum_{i=0}^{8} I_{i} .
$$

\section{Experimental results}

To determine the performance when repeating average filter, some comprehensive texture datasets are used in this section: the Outex [39], the UIUC [60], and the CUReT [61]. For all noisy textures, Gaussian noise is used.

\subsection{Dissimilarity metric method}

For comparing two textures, the LBP histograms of them must be compared. There are many methods for comparing two histograms, such as histogram intersection, log-likelihood ratio, and chi-square method [8]. In this paper, chi-square method is used for classification. Eq. (8) shows chi-square method. A test sample, $T$, is assigned to the class of model, $L$, that minimizes the chi-square value:

$$
D(T, L)=\sum_{i=1}^{N} \frac{\left(T_{i}-L_{i}\right)^{2}}{T_{i}+L_{i}}
$$

where $N$ is the number of bins of each histogram, and $T_{i}$ and $L_{i}$ are the values of the sample and the model image at the $i$ th bin, respectively. The nearest neighborhood (K-NN with $K=1$ ) classifier with the chi-square distance is used to measure the dissimilarity between two textures.

\subsection{Experimental results of the Outex dataset} The Outex dataset includes many test suites [39]. These suites have been collected under different illumination, rotation, and scaling conditions. Outex_TC_00010 (TC10) and two groups of Outex_TC_00012(TC12('t') and TC12('h')) are considered as some famous test suites in this dataset. They can be used for rotation-invariant tests. These two suites have the same 24 classes of textures, which are collected under three different illuminates (horizon, inca, and t184) and nine different rotation angles (00, 50, 100, 150, 300, 450, 600, 750, and 900).

There are 20 non-overlapping $128 * 128$ texture images for each class under each condition. Furthermore, in this paper, Outex_TC_00013 is used. This suite includes 68 texture classes with the size of $128 * 128$ and inca illumination. Figure 14 shows the 24 images of each class of Outex dataset. Table 2 compares the results of the proposed method and some state-ofthe-art noise-robust LBPs. This table and all other tables of this paper compare the results for noisy textures with $\mathrm{SNR}=100,30,15,10,5,3$, and 2 .

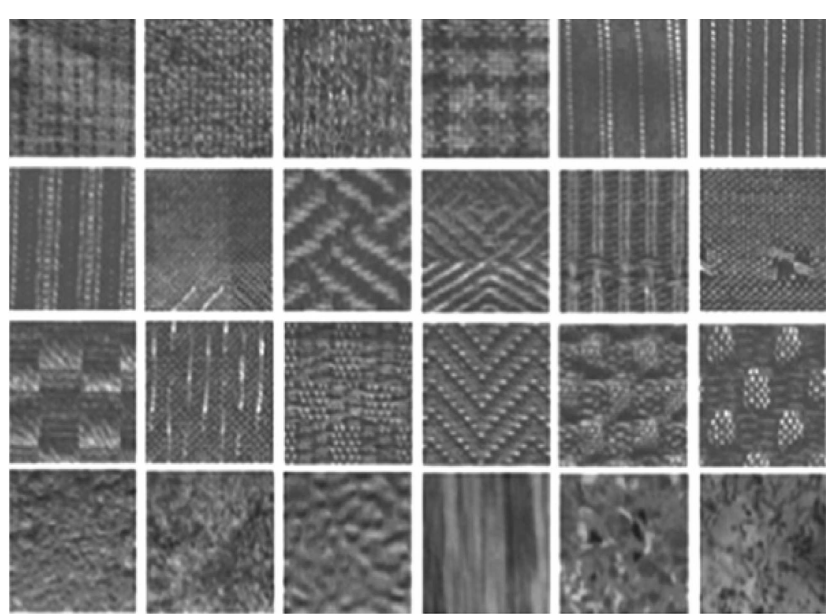

Figure 14. All 24 classes of Outex dataset.

Table 2 indicates that the RF_CLBP with optimal $n$ (number of repeats of the average filter), $R=3$, and $P=24$ provides the best performance for all values of SNR for TC10. Only for $\mathrm{SNR}=3$, the accuracy of BRINT1_CS_CM is slightly better than that of RF_CLBP. The table shows that RF_CLBP with $n=1$ is same as CRLBP. Therefore, for low noise values, the performance of RF_CLBP is same as that of CRLBP, but it is noticeably better than that of BRINT. On the other hand, for low SNR values, the accuracy of RF_CLBP with optimum value of $n$ is significantly higher than that of CRLBP, but the accuracy of BRINT is close to that of RF_CLBP. In other words, accuracy of the proposed method is higher than those of both CRLBP and BRINT and other types of noise-robust LBPs for low- and high-noise textures.

Tables 3 and 4 show the results of TC12('t') and TC12(' $h$ '). They also indicate that RF_CLBP outperforms all the other methods such as LTP, NTLBP, CRLBP, and BRINT. For very highly noisy textures, only BRINT method provides the accuracy near RF_CLBP. For low-noise textures, the accuracy of the proposed method is same as that of CRLBP. In both tables, the best accuracy for all SNR values is obtained by using RF_CLBP with optimum number of repeats of filter for $R=3$ and $P=24$. Only for $\mathrm{SNR}=$ 3 and 2 , in Table 3 , the accuracy of BRINT1 is slightly better than that of RF_CLBP.

Figures 15 and 16 show the accuracy of RF_CLBP for 2 suites of Outex dataset. In these figures, $R=1$ and $P=8$. Figure $15(\mathrm{a})$ shows that the best accuracies of RF_CLBP for TC10 when SNR $=30,10,5,3$, and 2 are around 3, 3, 4, 5, and 19, respectively. Figure 15(b) determines the performance of RF_CLBP for TC12( ' $t$ ') suites. According to this figure, the highest accuracies for $\mathrm{SNR}=30,10,5,3$, and 2 are obtained when $n$ (number of repeats of filter) is equal to 2, 2, 3, 4, and 25, respectively. Figure 16(a) determines the same trend for TC13 when $R=1$ and $P=8$. In this suite, 
Table 2. Classification rates for noisy Outex (TC10) textures using different SNR values.

\begin{tabular}{|c|c|c|c|c|c|c|c|}
\hline TC10 & $\mathrm{SNR}=100$ & $\mathrm{SNR}=30$ & $\mathrm{SNR}=15$ & $\mathrm{SNR}=10$ & $\mathrm{SNR}=5$ & $\mathrm{SNR}=3$ & $\mathrm{SNR}=2$ \\
\hline LBP & 81.09 & 74.66 & 64.66 & 48.78 & 22.40 & 10.63 & 5.55 \\
\hline CLBP_S/M/C $(R=1, P=8)$ & 97.55 & 96.07 & 93.65 & 88.39 & 51.98 & 17.79 & 8.28 \\
\hline $\operatorname{CRLBP}(R=1, P=8) \alpha=1$ & 98.10 & 97.40 & 96.43 & 94.92 & 83.67 & 46.09 & 17.26 \\
\hline $\operatorname{CRLBP}(R=1, P=8) \alpha=8$ & 97.92 & 97.37 & 95.91 & 93.46 & 74.11 & 32.03 & 13.25 \\
\hline $\operatorname{CRLBP}(R=3, P=24) \alpha=1$ & 99.43 & 99.35 & 98.93 & 97.76 & 92.27 & 71.96 & 29.12 \\
\hline $\operatorname{CRLBP}(R=3, P=24) \alpha=8$ & 99.27 & 98.96 & 98.26 & 96.12 & 85.81 & 64.23 & 20.18 \\
\hline NTLBP (MS9) & 98.65 & 96.12 & 88.85 & 80.23 & 51.09 & 30.34 & 12.78 \\
\hline LTP & 95.91 & 95.05 & 88.91 & 69.01 & 25.89 & 12.08 & 9.32 \\
\hline NRLBP (MS9) & 87.40 & 85.73 & 80.16 & 72.42 & 51.02 & 32.63 & 14.01 \\
\hline LBP (MS3) & 95.03 & 86.93 & 67.24 & 49.79 & 24.06 & 12.97 & 8.77 \\
\hline BRINT1_CS_CM (MS9) & 94.74 & 94.04 & 92.21 & 92.42 & 89.24 & 77.50 & 41.38 \\
\hline BRINT2_CS_CM (MS9) & 97.76 & 96.48 & 95.47 & 92.97 & 88.31 & 71.51 & 38.52 \\
\hline RF_CLBP $(R=1, P=8, n=1)$ & 97.14 & 96.80 & 95.63 & 93.46 & 79.87 & 44.27 & 15.00 \\
\hline RF_CLBP $(R=1, P=8, n=2)$ & 98.20 & 97.55 & 96.64 & 96.61 & 87.81 & 59.43 & 23.57 \\
\hline RF_CLBP $(R=1, P=8, n=$ opt $)$ & 98.49 & 98.33 & 97.97 & 96.82 & 91.90 & 76.49 & 40.12 \\
\hline RF_CLBP $(R=3, P=24, n=1)$ & 99.43 & 99.40 & 98.98 & 98.54 & 92.71 & 70.95 & 28.59 \\
\hline RF_CLBP $(R=3, P=24, n=2)$ & 99.19 & 99.22 & 98.96 & 98.75 & 94.58 & 76.16 & 31.30 \\
\hline RF_CLBP $(R=3, P=24, n=$ opt $)$ & 99.43 & 99.40 & 98.98 & 98.78 & 94.58 & 77.48 & 43.35 \\
\hline
\end{tabular}

Table 3. Classification rates for noisy Outex (TC12(' $\mathrm{t}$ ')) textures using different SNR values.

\begin{tabular}{|c|c|c|c|c|c|c|c|}
\hline TC12t & $\mathrm{SNR}=100$ & $\mathrm{SNR}=30$ & $\mathrm{SNR}=15$ & $\mathrm{SNR}=10$ & $\mathrm{SNR}=5$ & $\mathrm{SNR}=3$ & $\mathrm{SNR}=\mathbf{2}$ \\
\hline LBP & 71.27 & 64.56 & 53.38 & 42.18 & 20.86 & 9.88 & 5.63 \\
\hline CLBP_S/M/C $(R=1, P=8)$ & 90.93 & 87.41 & 84.05 & 79.77 & 48.73 & 18.19 & 8.19 \\
\hline $\operatorname{CRLBP}(R=1, P=8) \alpha=1$ & 93.84 & 91.71 & 90.56 & 87.29 & 75.39 & 42.89 & 13.98 \\
\hline $\operatorname{CRLBP}(R=1, P=8) \alpha=8$ & 92.57 & 91.06 & 89.44 & 83.47 & 65.16 & 27.41 & 28.02 \\
\hline $\operatorname{CRLBP}(R=3, P=24, N=46) \alpha=1$ & 97.34 & 97.08 & 96.50 & 94.91 & 87.97 & 66.34 & 27.01 \\
\hline $\operatorname{CRLBP}(R=3, P=24, N=46) \alpha=8$ & 96.46 & 96.41 & 95.28 & 92.69 & 79.61 & 52.92 & 21.54 \\
\hline NTLBP (MS9) & 92.15 & 89.35 & 83.77 & 74.47 & 49.84 & 31.27 & 12.01 \\
\hline LTP & 80.76 & 80.30 & 75.42 & 60.14 & 24.93 & 11.09 & 6.5 \\
\hline NRLBP (MS9) & 84.49 & 81.16 & 77.52 & 70.16 & 50.88 & 33.31 & 13.87 \\
\hline LBP (MS3) & 91.30 & 82.55 & 60.25 & 47.31 & 24.07 & 13.63 & 8.55 \\
\hline BRINT1_CS_CM (MS9) & 92.87 & 90.63 & 89.72 & 88.12 & 83.84 & 74.47 & 38.55 \\
\hline BRINT2_CS_CM (MS9) & 95.95 & 93.59 & 91.32 & 90.49 & 83.68 & 69.70 & 35.01 \\
\hline RF_CLBP $(R=1, P=8, n=1)$ & 92.38 & 91.67 & 90.69 & 87.25 & 75.26 & 44.68 & 14.44 \\
\hline RF_CLBP $(R=1, P=8, n=2)$ & 94.33 & 94.24 & 93.91 & 92.69 & 83.22 & 58.38 & 23.38 \\
\hline RF_CLBP $(R=1, P=8, n=$ opt $)$ & 94.33 & 94.24 & 94.12 & 92.80 & 87.01 & 71.53 & 34.47 \\
\hline RF_CLBP $(R=3, P=24, n=1)$ & 97.48 & 97.27 & 96.92 & 95.76 & 88.66 & 66.11 & 27.78 \\
\hline RF_CLBP $(R=3, P=24, n=2)$ & 97.50 & 97.45 & 96.67 & 95.44 & 91.18 & 70.51 & 34.21 \\
\hline RF_CLBP $(R=3, P=24, n=\mathrm{opt})$ & 97.62 & 97.45 & 96.92 & 95.76 & 91.50 & 74.33 & 38.41 \\
\hline
\end{tabular}


Table 4. Classification rates for noisy Outex (TC12(' $h$ ')) textures using different SNR values.

\begin{tabular}{|c|c|c|c|c|c|c|c|}
\hline TC12h & $\mathbf{S N R}=$ & $\mathbf{N R}=$ & $\mathrm{NR}=$ & $\mathbf{N R}=1$ & $\mathbf{N R}=$ & $\mathrm{NNR}=$ & $\mathbf{N R}=\mathbf{2}$ \\
\hline LBP & 68.06 & 64.03 & 55.58 & 45.02 & 21.37 & 10.37 & 6.09 \\
\hline CLBP_S/M/C $(R=1, P=8)$ & 92.52 & 90.53 & 86.90 & 81.78 & 50.60 & 17.87 & 7.31 \\
\hline $\operatorname{CRLBP}(R=1, P=8) \alpha=1$ & 94.12 & 92.66 & 90.44 & 88.40 & 77.45 & 43.17 & 12.08 \\
\hline $\operatorname{CRLBP}(R=1, P=8) \alpha=8$ & 93.91 & 93.50 & 90.35 & 85.93 & 70.56 & 29.56 & 22.50 \\
\hline $\operatorname{CRLBP}(R=3, P=24, N=46) \alpha=1$ & 96.44 & 97.04 & 96.57 & 95.49 & 88,06 & 63.22 & 23.88 \\
\hline $\operatorname{CRLBP}(R=3, P=24, N=46) \alpha=8$ & 95.63 & 95.88 & 94.98 & 93.59 & 79.68 & 42.88 & 19.87 \\
\hline NTLBP (MS9) & 94.35 & 90.81 & 84.95 & 75.49 & 47.04 & 30.38 & 12.04 \\
\hline LTP & 80.84 & 79.23 & 75.28 & 64.68 & 25.42 & 10.74 & 7.23 \\
\hline NRLBP (MS9) & 85.76 & 82.69 & 77.38 & 69.68 & 49.07 & 32.06 & 11.44 \\
\hline LBP (MS3) & 90.72 & 79.17 & 60.74 & 45.81 & 25.02 & 12.55 & 9.87 \\
\hline BRINT1_CS_CM (MS9) & 94.10 & 92.31 & 90.95 & 89.84 & 85.83 & 76.04 & 34.15 \\
\hline BRINT2_CS_CM (MS9) & 96.92 & 95.14 & 93.66 & 92.29 & 84.77 & 71.02 & 32.56 \\
\hline RF_CLBP $(R=1, P=8, n=1)$ & 93.36 & 92.80 & 91.19 & 89.00 & 76.02 & 45.67 & 13.96 \\
\hline RF_CLBP $(R=1, P=8, n=2)$ & 95.83 & 95.58 & 94.91 & 94.95 & 84.70 & 59.24 & 23.33 \\
\hline RF_CLBP $(R=1, P=8, n=$ opt $)$ & 95.83 & 95.58 & 94.91 & 94.95 & 89.54 & 71.27 & 33.59 \\
\hline RF_CLBP $(R=3, P=24, n=1)$ & 97.13 & 97.36 & 96.62 & 95.07 & 88.63 & 66.13 & 25.69 \\
\hline RF_CLBP $(R=3, P=24, n=2)$ & 97.66 & 97.57 & 97.11 & 96.11 & 91.16 & 70.81 & 32.50 \\
\hline RF_CLBP $(R=3, P=24, n=$ opt $)$ & 97.66 & 97.57 & 97.11 & 96.11 & 91.16 & 76.12 & 34.17 \\
\hline
\end{tabular}

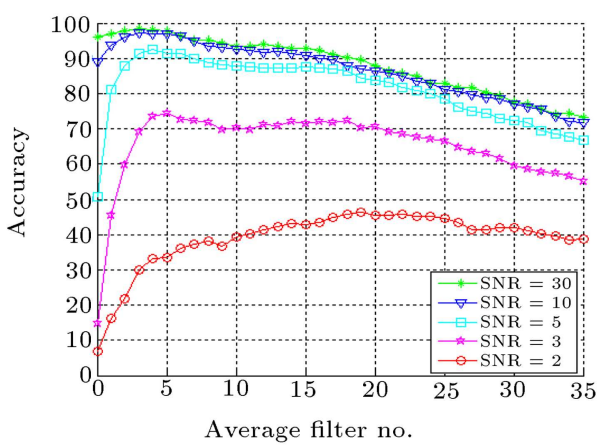

(a)

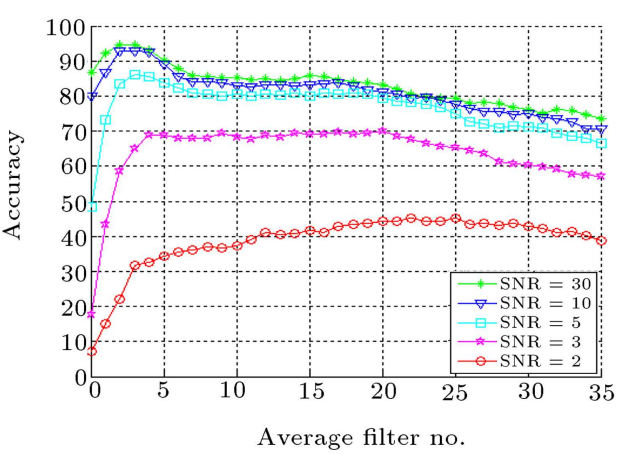

(b)

Figure 15. The performance of RF_CLBP $(R=1, P=8)$ for different numbers of repeats of average filter for noisy Outex Dataset: (a) TC10 and (b) TC12(t).

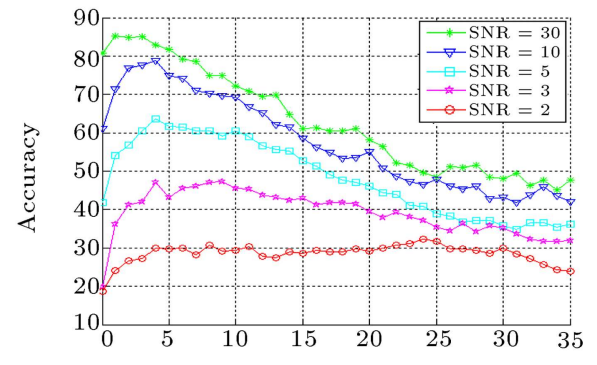

Average filter no.

(a)

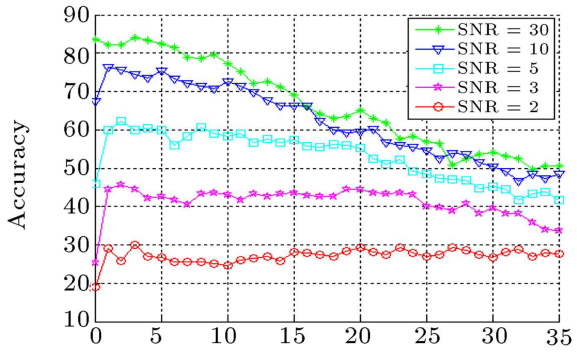

Average filter no.

(b)

Figure 16. The performance of RF_CLBP for different numbers of repeats of average filter for noisy Outex (TC13): (a) $R=1, P=8$ and (b) $R=3, P=24$. 
when $\mathrm{SNR}=30$, the highest accuracy is obtained for $n=1$, but it is 4 for $\mathrm{SNR}=10$. Furthermore, the best performances for $\mathrm{SNR}=5,3$, and 2 are achieved when $n=4,4$, and, 19 respectively. All of the plots in Figures 15 and 16(a) use RF_CLBP with $R=1$ and $P=8$. The performance of RF_CLBP improves for larger neighborhoods with the similar trend. The performance of RF_CLBP for $R=3$ and $P=24$ is shown in Figure 16(b). This plot indicates that the best numbers of repeats for $\mathrm{SNR}=30,10,5$, 3 , and 2 are achieved when $n$ is $3,1,2,2$, and 3 , respectively. Figures 15 and 16 show that for large values of SNR, the best performance is achieved after 1,2 , or at $\max , 3$ times of using average filter while for high noisy textures, it is necessary to repeat the filter more times. Furthermore, after some special number of using average filter, the accuracy decreases. However, this reduction is moderate for large neighborhood.

Some methods such as GLCM that use cooccurrence matrix are not rotation-invariant and they are sensitive to noise. Therefore, if they are used for these textures, the classification accuracy decreases significantly [55].

\subsection{Experimental results of the UIUC dataset}

The UIUC dataset [60] contains 25 classes with 40 images in each class. Resolution of each texture image is $640 * 480$. Figure 17 shows one image for each class of UIUC dataset. For implementation, each time, $N=20$ images of each class are selected randomly for train and the rest of them $(40-N)$ are used for test. This operation is run 100 times and the average of the results is shown in Table 5. This table compares the accuracy of the proposed method with those of LBP, LTP, CLBP, and CRLBP. In this table, for all values of SNR, the highest performance is achieved by using RF_CLBP when $R=3$ and $P=24$ for optimum value of $n$. Also,
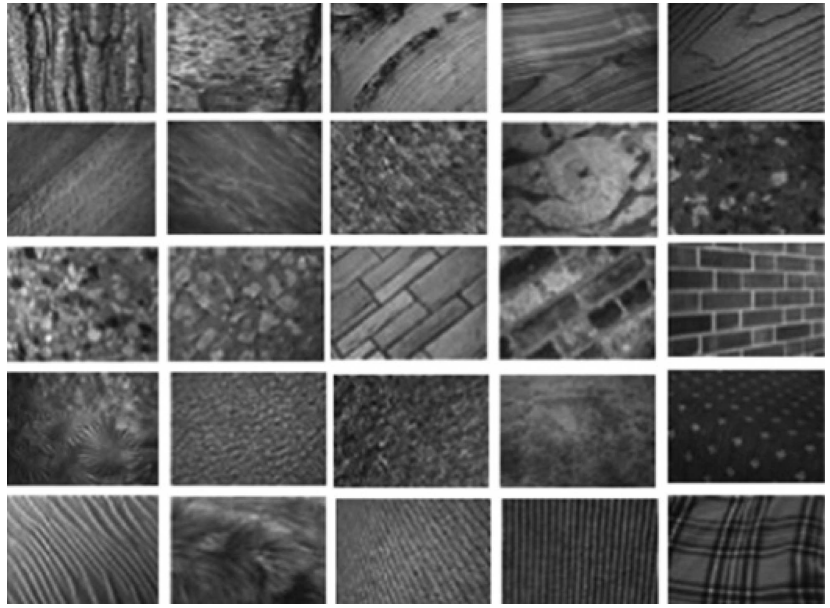

Figure 17. All 25 classes of UIUC dataset.

RF_CLBP with $R=1$ and $P=8$ has the second best performance in this table.

As it is mentioned before, CRLBP is same as RF_CLBP with $n=1$. Therefore, these two methods are almost the same considering accuracy for lownoise textures; however, for highly noisy textures, the accuracy of RF_CLBP is higher by far than those of all CRLBP and all other LBP variants such as LTP, LBP, and CLBP. Also, the results of LBP-based and nonLBP methods when used for UIUC textures are shown in Table 6 in the next sections.

\subsection{Experimental results of the CUReT dataset}

The CUReT dataset [61] contains 61 classes of textures. They are shown in Figure 18. These images are captured in different viewpoints and illumination orientations. For each class, 92 images are selected from the images that have a viewing angle of less than $60^{\circ}$. Each time, $N=46$ images are randomly

Table 5. Classification rates for noisy UIUC textures using different SNR values. $(N=20)$.

\begin{tabular}{|c|c|c|c|c|c|c|c|}
\hline UIUC & $\mathrm{SNR}=1$ & $\mathrm{NR}=3$ & $\mathbf{N R}=$ & $\mathrm{NR}=$ & $\mathbf{N R}=$ & $\mathrm{NR}=$ & $\mathbf{N R}=\mathbf{2}$ \\
\hline $\operatorname{LBP}(R=1)$ & 54.40 & 57.30 & 54.35 & 48.16 & 44.08 & 41.15 & 42.18 \\
\hline $\operatorname{LTP}(R=1)$ & 76.88 & 77.44 & 73.00 & 66.14 & 62.47 & 60.24 & 59.12 \\
\hline CLBP_S/M/C $(R=1, P=8, N=20)$ & 87.42 & 87.68 & 84.68 & 81.44 & 72.48 & 71.76 & 66.47 \\
\hline CLBP_S/M $/ \mathrm{C}(R=3, P=24, N=20)$ & 90.74 & 90.38 & 87.56 & 81.64 & 74.54 & 73.85 & 68.23 \\
\hline $\operatorname{CRLBP}(R=3, P=24, N=20) \alpha=1$ & 93.49 & 93.08 & 92.74 & 88.57 & 80.20 & 76.55 & 70.55 \\
\hline $\operatorname{CRLBP}(R=3, P=24, N=20) \alpha=8$ & 92.51 & 92.28 & 91.11 & 85.90 & 77.16 & 73.12 & 68.85 \\
\hline $\operatorname{RF} \_$CLBP $(R=1, P=8, n=1)$ & 89.55 & 89.38 & 88.20 & 87.91 & 81.78 & 75.12 & 68.51 \\
\hline RF_CLBP $(R=1, P=8, n=2)$ & 89.84 & 89.77 & 90.00 & 89.41 & 85.31 & 78.03 & 70.24 \\
\hline RF_CLBP $(R=1, P=8, n=\mathrm{opt})$ & 90.21 & 90.03 & 90.17 & 90.19 & 87.13 & 82.72 & 75.70 \\
\hline RF_CLBP $(R=3, P=24, n=1)$ & 93.73 & 93.15 & 92.82 & 89.81 & 81.27 & 76.93 & 70.40 \\
\hline RF_CLBP $(R=3, P=24, n=2)$ & 94.52 & 94.44 & 94.64 & 94.10 & 89.87 & 81.85 & 75.01 \\
\hline $\operatorname{RF} \_C L B P(R=3, P=24, n=o p t)$ & 94.82 & 94.80 & 94.64 & 94.28 & 92.32 & 87.19 & 82.77 \\
\hline
\end{tabular}


Table 6. Classification rates for 3 datasets of textures.

\begin{tabular}{lccc}
\hline \multicolumn{1}{c}{ Methods } & Outex & UIUC $(\boldsymbol{N}=\mathbf{2 0})$ & CUReT $(\boldsymbol{N}=\mathbf{4 6})$ \\
\hline LBP & 81.19 & 54.51 & 77.54 \\
CLBP_S/M/C $(R=1, P=8)$ & 97.66 & 87.52 & 95.56 \\
CRLBP $(R=1, P=8) \alpha=1$ & 98.12 & 93.54 & 94.70 \\
CRLBP $(R=1, P=8) \alpha=8$ & 97.97 & 92.58 & 95.58 \\
CRLBP $(R=3, P=24) \alpha=1$ & 99.42 & 93.65 & 96.21 \\
CRLBP $(R=3, P=24) \alpha=8$ & 99.34 & 92.57 & 96.42 \\
NTLBP $($ MS9 $)$ & 98.77 & - & 91.64 \\
LTP & 95.98 & 76.94 & 84.92 \\
BRINT1_CS_CM $($ MS9) & 94.80 & - & $\mathbf{9 6 . 8 5}$ \\
BRINT2_CS_CM $(\mathrm{MS} 9)$ & 97.87 & - & 96.80 \\
RF_CLBP $(R=1, P=8, n=1)$ & 97.24 & 89.57 & 96.07 \\
RF_CLBP $(R=1, P=8, n=2)$ & 98.21 & 89.88 & 95.28 \\
RF_CLBP $(R=1, P=8, n=$ opt $)$ & 98.54 & 90.26 & 96.06 \\
RF_CLBP $(R=3, P=24, n=1)$ & 99.44 & 93.75 & 95.99 \\
RF_CLBP $(R=3, P=24, n=2)$ & 99.21 & 94.62 & 95.15 \\
RF_CLBP $(R=3, P=24, n=$ opt $)$ & $\mathbf{9 9 . 4 5}$ & 94.83 & 96.11 \\
GLCM & 83.02 & 75.67 & - \\
GLCM, pyramid decomposition & 84.61 & 73.99 & - \\
GLCM, Gaussian smoothing & 81.79 & 81.88 & - \\
Multi scale GLCM & 89.40 & 81.76 & - \\
Gabor & 84.90 & 69.90 & 87.50 \\
VZ_Joint & 98.51 & 80.20 & 96.59 \\
VZ_MR8 & 94.06 & 92.14 & 95.75 \\
Lazebnik $(H+L)(S+R)$ & - & 95.42 & \\
HA+SIFT & - & $\mathbf{9 7 . 5 0}$ & \\
\hline
\end{tabular}

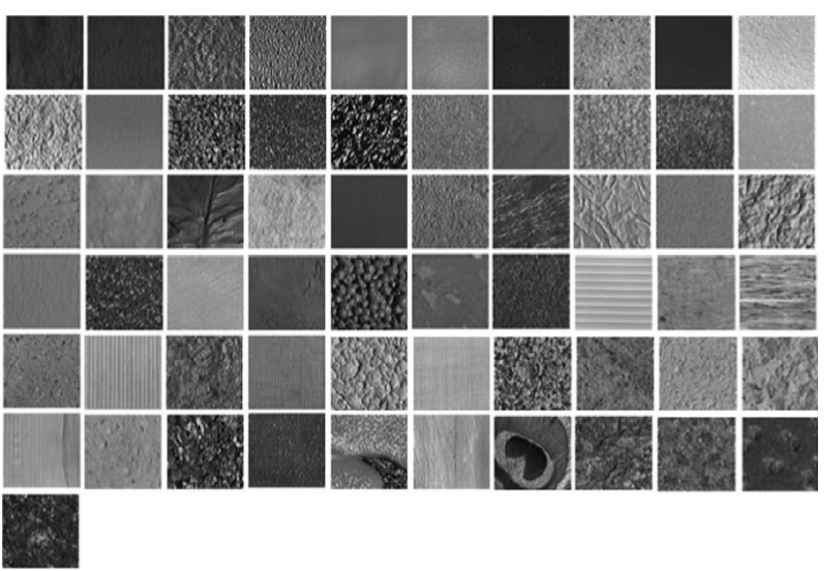

Figure 18. All 61 classes of CUReT dataset.

chosen for train from each class. The remaining $(92-N)$ images are used as test samples. The average classification rates over 100 random tests are shown in Table 7 .

In Table 7 , the comparison between the proposed methods and some LBP noise-resistant methods such as BRINT, CRLBP, LTP, DLBP, and NTLBP is illustrated. The wavelet transform features (DBWP) [23] and Circular Gaussian MRFs (ACGMRF) [12] are other two methods that are illustrated in the table. The results of some Gabor filter methods such as
TGF [17], CGF [18], and NGF [19] are shown in this table. Furthermore, DLBP+NGF is a method that uses Dominant LBP (DLBP) [19] with normal Gabor filter. It can be seen in the table that for all SNR $<100$ values, the proposed method provides the best accuracy. Only for SNR $=100$, the performance of BRINT1_CS_CM (MS9) is slightly higher than that of RF_CLBP. Similar to Outex dataset, in CUReT dataset, the accuracy of RF_CLBP is slightly better than that of CRLBP for low noise. Also, this accuracy is slightly better than that of BRINT for highly noisy textures. However, for high SNR values, accuracy of the proposed method is significantly higher than that of BRINT. On the other hand, this performance is noticeably more accurate than that of CRLBP for very noisy textures.

As it is mentioned in some papers [55], the performance of Gabor filter and co-occurrence matrix methods significantly declines for noisy textures. Table 7 shows some of these results.

\subsection{Comparison for normal textures (without noise)}

In this section, the comparison between accuracy of the proposed method and accuracy of some LBPbased, co-occurrence, Gabor Filter, patch based, and general descriptor approaches is shown. Table 6 shows 
Table 7. Classification rates for noisy CUReT textures using different SNR values $(N=46)$.

\begin{tabular}{|c|c|c|c|c|c|c|c|}
\hline CUReT & $\mathrm{SNR}=1$ & $\mathbf{N R}=$ & $\mathbf{N R}=$ & $\mathbf{N R}=1$ & $\mathbf{N R}=$ & $\mathrm{NR}=$ & $\mathrm{NR}=\mathbf{2}$ \\
\hline LBP & 77.47 & 73.25 & 67.50 & 62.72 & 50.25 & 39.72 & 25.78 \\
\hline CLBP_S/M $/ \mathrm{C}(R=1, P=8, N=46)$ & 95.49 & 94.42 & 91.97 & 88.11 & 77.91 & 66.30 & 50.34 \\
\hline CLBP_S/M/C $(R=3, P=24, N=46)$ & 95.51 & 95.87 & 87.23 & 72.77 & 61.35 & 57.77 & 54.20 \\
\hline $\operatorname{CRLBP}(R=3, P=24, N=46) \alpha=1$ & 96.06 & 95.90 & 93.56 & 85.58 & 79.67 & 74.55 & 61.22 \\
\hline $\operatorname{CRLBP}(R=3, P=24, N=46) \alpha=8$ & 96.34 & 96.18 & 92.30 & 82.88 & 74.97 & 69.98 & 57.55 \\
\hline NTLBP (MS9) & 91.56 & 85.99 & 78.98 & 74.90 & 65.74 & 56.31 & 39.45 \\
\hline LTP & 84.82 & 84.06 & 79.60 & 72.89 & 57.62 & 47.13 & 37.27 \\
\hline LTP (MS9) & 92.22 & 90.15 & 86.66 & 84.55 & 77.48 & 70.67 & 41.05 \\
\hline BRINT1_CS_CM (MS9) & 96.81 & 95.39 & 93.69 & 90.92 & 86.11 & 80.45 & 64.87 \\
\hline BRINT2_CS_CM (MS9) & 96.78 & 94.90 & 92.83 & 90.46 & 84.48 & 78.33 & 63.21 \\
\hline RF_CLBP $(R=1, P=8, n=1)$ & 96.01 & 95.83 & 95.35 & 92.77 & 83.99 & 74.31 & 58.05 \\
\hline RF_CLBP $(R=1, P=8, n=2)$ & 95.22 & 95.94 & 96.24 & 95.21 & 88.05 & 80.66 & 63.63 \\
\hline RF_CLBP $(R=1, P=8, n=$ opt $)$ & 96.01 & 95.94 & 96.24 & 95.21 & 87.12 & 81.99 & 64.63 \\
\hline RF_CLBP $(R=3, P=24, n=1)$ & 95.97 & 96.14 & 95.24 & 95.90 & 86.56 & 74.71 & 60.80 \\
\hline RF_CLBP $(R=3, P=24, n=2)$ & 95.14 & 95.97 & 95.73 & 95.20 & 87.44 & 77.12 & 64.91 \\
\hline RF_CLBP $(R=3, P=24, n=$ opt $)$ & 96.07 & 96.19 & 96.24 & 95.90 & 90.72 & 81.93 & 65.63 \\
\hline DBWP & 88.37 & 85.33 & 80.38 & 71.40 & 63.40 & - & - \\
\hline TGF & 60.79 & 62.69 & 51.80 & 43.67 & 46.41 & - & - \\
\hline CGF & 52.94 & 53.39 & 52.36 & 46.39 & 46.74 & - & - \\
\hline ACGMRF & 66.90 & 57.54 & 51.36 & 47.05 & 47.08 & - & - \\
\hline NGF & 47.86 & 45.49 & 43.14 & 41.63 & 40.06 & - & - \\
\hline DLBP & 87.82 & 85.28 & 79.44 & 68.73 & 47.72 & - & - \\
\hline $\mathrm{DLBP}+\mathrm{NGF}$ & 96.17 & 95.78 & 92.81 & 86.06 & 71.28 & - & - \\
\hline
\end{tabular}

the results of the proposed method and some other methods for 3 datasets. In this table, the methods which are related to 4 groups of texture classification are shown: GLCM method, a Gabor method, two scale-invariant and patch-based methods of VZ_Joint and VZ_MR8 [62,63], and general descriptor methods that include Lazebnik [64] and SIFT [34]. This table shows the results of texture classification without noise. If noise is added to the texture, the accuracy of classification of some methods considerably decreases. According to this table, the best accuracy for Outext is obtained by the proposed method. HA+SIFT provides the best accuracy for UIUC. However, the SIFT-based methods are very time consuming approaches. For CUReT textures, BRINT1_CS_CM (MS9) achieves the best classification accuracy. This method uses multi resolution parameters for an especial version of LBP. MS9 refers to the 9 different combinations of $\mathrm{R}$ and $\mathrm{P}$ (LBP parameters). This table shows that the accuracy of the proposed method is noticeably higher than that of Gabor and co-occurrence based methods (GLCM).

General descriptors such as SIFT and Lazebnik [64] achieve high accuracy only for UIUC textures. These types of textures have large numbers of key points; therefore, their accuracy is high. The accuracy of general descriptors such as SIFT decreases for some textures such as CUReT. This dataset contains some textures that have low contrast. Therefore, number of key points of some CUReT textures is very low. For the default threshold of SIFT, the number of key points of some CUReT textures is zero.

\subsection{Comparison with other average filtering methods}

In this paper, it is shown that repeating the mean filter decreases the noise and increases the classification accuracy of textures. A $3 \times 3$ square mean filter is used in the proposed method. The size of window must be small for better description of edge details and avoidance of corruption of edge and details of textures. The smallest size of window that is symmetric is $3 \times 3$. Thus, it was selected for this paper. If a larger size had been selected, it would have corrupted the edge and local details of textures. There are some other average filtering methods that remove noise from image. Nagao [65] and improved Nagao [66] are two types of these methods. These methods preserve edge and textures details better than simple mean method. If 
they had been used instead of the proposed method, the classification accuracy would have slightly increased, but the computational time would have been very high. In this section, the computational times of the proposed method and these two methods are compared.

Table 8 determines that the proposed fast mean method increases the speed of mean square method around 30 times. Table 9 shows the time of feature extraction for some methods [24]. This table determines the time of feature extraction for 2 datasets. According to this table, the proposed method (RF_CLBP with the proposed fast mean) is considerably faster than other methods.

Some average spatial filters have been proposed for noise reduction. Nagao [65] is one of these methods. The idea of Nagao filter is as follows: firstly, a rectangle template around the central pixel is rotated and the position of minimum variance as the default direction template is chosen; then, the value of the central pixel is replaced by the average value of the default template. Such processes are iterated until the pixels do not vary. The performance of Nagao filter depends on the shape of its template. The computational time of Nagao filter is also very high. The effect of Nagao filter is not satisfying for its mean filter style [66]. Therefore, Zhang and Wu proposed the improved Nagao filter or window-length Nagao filter (AWN) method [66]. They

Table 8. Average time of mean and the proposed fast mean for a texture (msec).

\begin{tabular}{lccc}
\hline & Mean & $\begin{array}{c}\text { Proposed } \\
\text { fast mean }\end{array}$ & Ratio \\
\hline UIUC & 2562.5 & 72.6 & 35.29 \\
Outex (TC10) & 78.71 & 2.57 & 30.62 \\
CUReT & 52.33 & 1.84 & 28.44 \\
\hline
\end{tabular}

showed that by using median filter instead of mean filter and some techniques, the edge and some other details of image were preserved and better performance than that of Nagao method could be obtained.

Both Nagao and AWN methods can preserve edge and textures details better than simple mean method. But, the computational times of theme are considerably higher than that of mean method. As it is shown in Table 8, the proposed fast mean method increases the speed of square mean method around 30 times in comparison with the traditional mean filter. Nagao filter is slower than simple mean filter at least 10 times. In other words, speed of the proposed fast mean method is higher than that of Nagao around 300 times. For improved Nagao (AWN) method, median or complex templates are used; therefore, the speed of AWN is lower than that of Nagao and the ratio is higher than 300 for AWN method.

\subsection{Comparison with other methods considering LBP specifications}

Table 10 compares specifications of some texture features extraction methods. In this paper, the LBP with riu 2 mapping is used. It is shown in the table that local binary pattern (riu2) is a rotation-invariant and grayscale-invariant method [67]. The computational time of this method is low and it extracts a small number of features. However, LBP is not scale-invariant and sensitive to noise. The proposed method and some advanced LBPs [55] provide noise robustness for LBP. It is a simple method that can be combined with other methods and it has achieved high performance in classification results for many kinds of texture datasets [39]. It has been extensively exploited in many applications, e.g. face image analysis, image and video retrieval, environment modeling, visual inspection, mo-

Table 9. Computational time (sec) required for feature extraction.

\begin{tabular}{cccccccc}
\hline Dataset & LBP & CLBP & $\begin{array}{c}\text { RF_CLBP } \\
(\boldsymbol{n}=\mathbf{1})\end{array}$ & $\begin{array}{c}\text { RF_CLBP } \\
\text { fast mean } \\
(\boldsymbol{n}=\mathbf{1})\end{array}$ & Gabor & GLCM & $\begin{array}{c}\text { Multi } \\
\text { scale } \\
\text { GLCM }\end{array}$ \\
\hline UIUC & 24.95 & 37.20 & 284.50 & 44.70 & 1371.59 & 39.35 & 183.10 \\
Outex & 1.89 & 4.93 & 21.56 & 5.45 & 297.94 & 41.66 & 180.49 \\
\hline
\end{tabular}

Table 10. Comparison between LBP and other approaches.

\begin{tabular}{lccccccc}
\hline & Simple LBP & LBP $^{\text {riu2 }}$ & CLBP $^{\text {riuz }}$ & Proposed & SIFT & GLCM & Gabor \\
\hline Gray-scale-invariant & Yes & Yes & Yes & Yes & Yes & No & Yes \\
Rotate-invariant & No & Yes & Yes & Yes & Yes & No & Yes \\
Scale-invariant & No & No & No & No & Yes & No & Yes \\
Noise robustness & No & No & No & Yes & Yes & No & No \\
Computational time & Medium & Low & Low & Low & Very high & Medium & High \\
Number of features & High & Low & Medium & Medium & High & Medium & Low \\
\hline
\end{tabular}


tion analysis, biomedical and aerial image analysis, and remote sensing [68]. Some methods such as [69] extract features which are robust not only to the noise but also to the change of noise.

\section{Conclusion}

In this paper, it is shown that repeating average filter for noisy texture increases the classification accuracy significantly. The more value of noise, the more number of repeats of average filter should be used for noisy textures. The optimal value for repeating the average filter is obtained when variance of texture reaches the lowest values or the change of it is smaller than a threshold value. The performance of the proposed method is better than that of some novel and advanced noise-robust LBP methods. Some state-of-the-art methods such as CRLBP provide good performance only for highly SNR noisy textures. On the other hand, some advanced methods such as BRINT provide high accuracy for low SNR values. The proposed method provides the best performance for both low and high SNR values. Furthermore, by using the fast technique that is proposed in this paper, the speed of applying average filter increases significantly. Therefore, the speed of the proposed method is by far higher than those of almost all the noise-robust LBP methods that are used in this paper. The proposed method is used as a preprocessing operation for noisy textures. After this pre-processing operation, CLBP is used for feature extraction. It is possible to use any other types of feature extraction methods to extract features of textures.

\section{References}

1. Cohen, F.S., Fan, Z. and Attali, S. "Automated inspection of textile fabrics using Textural models", IEEE Transactions on Pattern Analysis and Machine Intelligence, 13(8), pp. 803-808 (1991).

2. Tajeripour, F., Kabir, E. and Sheikhi, A. "Fabric defect detection using modified local binary patterns", EURASIP Journal on Advances in Signal Processing, 8, pp. 1-12 (2008).

3. Ji, Q., Engel, J. and Craine, E. "Texture analysis for classification of cervix lesions", IEEE Transactions on Medical Imaging, 19(11), pp. 1144-1149 (2000).

4. Anys, H. and He, D.C. "Evaluation of textural and multi polarization radar features for crop classification", IEEE Transactions on Geoscience and Remote Sensing, 33(5), pp.1170-1181 (1995).

5. Zhang, B., Gao, Y., Zhao, S. and Liu, J. "Local derivative patterns versus local binary patterns: face recognition with high-order local patterns descriptor", IEEE Transactions on Image Processing, 19(2), pp. 533-544 (2010).
6. Murala, S., Maheshwari, R.P. and Subramanian, R.B. "Local tetra patterns: a new feature descriptor for content-based image retrieval", IEEE Transactions on Image Processing, 21(5), pp. 2874-2886 (2012).

7. Haralik, R.M., Shanmugam, K. and Dinstein, I. "Texture features for image classification", IEEE Transactions on Systems, Man and Cybernetics, 3(6), pp. 610621 (1973).

8. Ojala, T., Pietikainen, M. and Maenpa, T.T. "Multiresolution gray-scale and rotation Invariant texture classification with local binary patterns", IEEE Transactions on Pattern Analysis and Machine Intelligence, 24(7), pp. 971-987 (2002).

9. Chen, J.L. and Kundu, A. "Rotation and gray scale transform invariant texture identification using wavelet decomposition and hidden Markov model", IEEE Transactions on Pattern Analysis and Machine Intelligence, 16(2), pp. 208-214 (1994).

10. Campisi, P., Neri, A., Panci, C. and Scarano, G. "Robust rotation-invariant texture classification using a model based approach", IEEE Transactions on Image Processing, 13(6), pp. 782-791 (2004).

11. Kashyap, R.L. and Khotanzad, A. "A model-based method for rotation invariant texture classification", IEEE Transactions on Pattern Analysis and Machine Intelligence, 8(4), pp. 472-481 (1986).

12. Deng, H. and Clausi, D.A. "Gaussian MRF rotationinvariant features for image classification", IEEE Trans. Pattern Anal. Mach. Intell., 26(7), pp. 951-955 (2004).

13. Chellappa, R. and Chatterjee, S., "Classification of textures using Gaussian Markov random fields", IEEE Trans. Acoust., Speech, Signal Process., ASSP-33, 4, pp. 959-963 (1985).

14. Eichmann, G. and Kasparis, T. "Topologically invariant texture descriptors", Computer Vision, Graphics and Image Processing, 41(3), pp. 267-281 (1988).

15. Lam, W.K. and Li, C. "Rotation texture classification by improved iterative morphological decomposition", IEEE Proceedings Vision, Image and Signal Processing, 144(3), pp. 171-179 (1997).

16. Daugman, J.G. "Uncertainty relation for resolution in space, spatial frequency, and orientation optimized by two dimensional visual cortical filters", Journal of the Optical Society of America, pp. 1160-1169 (1985).

17. Bovik, A.C., Clark, M. and Geisler, W.S. "Multichannel texture analysis using localized spatial filters", IEEE Trans. Pattern Anal. Mach. Intell., 12(1), pp. 55-73 (1990).

18. Haley, G.M. and Manjunath, B.S. "Rotation-invariant texture classification using a complete space-frequency model", IEEE Trans. Image Process., 8(2), pp. 255269 (1999).

19. Liao, S., Law, M.W.K. and Chung, A.C.S. "Dominant local binary patterns for texture classification", IEEE Trans. on Image Processing, 18(5), pp. 1107-1118 (2009). 
20. Arof, H. and Deravi, F. "Circular neighborhood and1DDFT features for texture classification and segmentation", IEEE Proceedings Vision, Image, and Signal Processing, 145(3), pp. 167-172 (1998)

21. Kim, S. "Texture classification using rotation wavelet filters", IEEE Transactions on Systems, Man and Cybernetics, Part A: Systems and Humans, 30(6), pp. 847-852 (2000).

22. Kokare, M., Biswas, P.K. and Chatterji, B.N. "Rotation-invariant texture image retrieval using rotation complex wavelet filters", IEEE Transactions on Systems, Man and Cybernetics, Part B: Cybernetics, 36(6), pp. 1273-1282 (2006).

23. Mallat, S.G. "A theory for multiresolution signal decomposition: The wavelet representation", IEEE Trans. Pattern Anal. Mach. Intell., 11(7), pp. 674-693 (1989).

24. Siqueira, F.R., Schwartz, W.R. and Pedrini, H. "Multiscale gray level co-occurrence matrices for texture description", Neurocomputing, pp. 336-345 (2013).

25. Clausi, D. and Jernigan, M. "A fast method to determine co-occurrence texture features", IEEE Transactions on Geoscience and Remote Sensing, 36(1), pp. 298-300 (1998).

26. Gelzinis, A., Verikas, A. and Bacauskiene, M. "Increasing the discrimination power of the co-occurrence matrix-based features", Pattern Recognition, 40(9), pp. 2367-2372 (2007).

27. Walker, R., Jackway, P. and Longstaff, D. "Genetic algorithm optimization of adaptive multi-scale GLCM features", International Journal of Pattern Recognition and Artificial Intelligence, 17(1), pp. 17-39 (2003).

28. Benco, M. and Hudec, R. "Novel method for color textures features extraction based on GLCM", Radio Engineering, 4(16), pp. 64-67 (2007).

29. Hu, Y. "Unsupervised texture classification by combining multi-scale features and K-means classifier", In Chinese Conference on Pattern Recognition, pp. 1-5 (2009).

30. Pacifici, F. and Chini, M. "Urban land-use multi-scale textural analysis", In IEEE International Geoscience and Remote Sensing Symposium, pp. 342-345 (2008).

31. Rakwatin, P., Longepe, N., Isoguchi, O., Shimada, M. and Uryu, Y. "Mapping tropical forest using ALOS PALSAR $50 \mathrm{~m}$ resolution data with multiscale GLCM analysis", In IEEE International Geoscience and Remote Sensing Symposium, pp. 1234-1237 (2010).

32. Nguyen-Duc, H., Do-Hong, T., Le-Tien, T. and BuiThu, C. "A new descriptor for image retrieval using contourlet co-occurrence", In Communications and Electronics (ICCE), Third International Conference, pp. 169-174 (2010).

33. Do, M., Vetterli, M., The Contourlet Transform: 552 "An efficient directional multi resolution image representation", IEEE Transactions on Image Processing, 14(12), pp. 2091-2106.
34. Lowe, D.G. "Distinctive image features from scaleinvariant keypoints", International Journal of Computer Vision, 60, pp. 91-110 (2004)

35. Bay, H., Tuytelaars, T. and Van Gool, L. "SURF: speeded-up robust features", In European Conference on Computer Vision, pp. 346-359 (2006).

36. Dalai, N., Triggs, B., Rhone-Alps, I. and Montbonnot, F. "Histograms of oriented gradients for human detection", In IEEE Conference on Computer Vision and Pattern Recognition, pp. 886-893 (2005).

37. Mikolajczyk, K. and Schmid, C. "A performance evaluation of local descriptors", IEEE Transactions on Pattern Analysis and Machine Intelligence, 27(10), pp.1615 -1630 (2005).

38. Ojala, T., Pietikäinen, M. and Harwood, D.A. "Comparative Study of texture measures with classification based on feature distributions", Pattern Recognition, 29(1), pp. 51-59 (1996).

39. Ojala, T., Mäenpää, T., Pietikäinen, M., Viertola, J., Kyllönen, J. and Huovinen, S. "Outex - new framework for empirical evaluation of texture analysis algorithm", in Proc. International Conference on Pattern Recognition, pp. 701-706 (2002).

40. Ahonen, T., Hadid, A. and Pietikäinen, M. "Face recognition with local binary patterns: application to face recognition", IEEE Trans. on Pattern Analysis and Machine Intelligence, 28(12), pp. 2037-2041 (2006).

41. Zhao, G. and Pietikäinen, M. "Dynamic texture recognition using local binary patterns with an application to facial expressions", IEEE Trans. on Pattern Analysis and Machine Intelligence, 27(6), pp. 915-928 (2007).

42. Huang, X., Li, S.Z. and Wang, Y. "Shape localization based on statistical method using extended local binary patterns", In Proc. International Conference on Image and Graphics, pp. 184-187 (2004).

43. Zabih, R. and Wood, J. "Non-parametric local transforms for computing visual correspondence", In Proc. Euro. Conf. Comput. Vis., pp. 151-158 (1994).

44. Ojala, T. "Nonparametric texture analysis using simple spatial operators, with applications in visual inspection", Acta Universitatis Oulue nsis, C 105 (1997).

45. Pietikäinen, M., Ojala, T. and Xu, Z. "Rotationinvariant texture classification using feature distributions", Pattern Recognition, 33(1), pp. 43-52 (2000).

46. Jin, H., Liu, Q., Lu, H. and Tong, X. "Face detection using improved LBP under Bayesian framework", In Proceedings of the 3rd International Conference on Image and Graphics, ICIG 2004, pp. 306-309 (2004).

47. Tan, X. and Triggs, B. "Enhanced local texture feature sets for face recognition under difficult lighting conditions", In Proc. International Workshop on Analysis and Modeling of Faces and Gestures, pp. 168-182 (2007). 
48. Iakovidis, D.K., Keramidas, E.G. and Maroulis, D. "Fuzzy local binary patterns for ultra sound texture characterization", In Proceedings of the 5th International Conference on Image Analysis and Recognition, ICIAR 2008, 5112 of Lecture Notes in Computer Science, Povoa de Varzim, Portugal, pp. 750-759 (2008).

49. Ahonen, T. and Pietikainen, M. "Soft histograms for local binary patterns", In Proceedings of the Finnish Signal Processing Symposium, FINSIG 2007, 1, Oulu, Finland, pp. 1-4 (2007).

50. Hafiane, A., Seetharaman, G. and Zavidovique, B. "Median binary pattern for textures classification", In Proceedings of the 4thInternational Conference, ICIAR 2007, 4633 of Lecture Notes in Computer Science, Montreal, Canada, pp. 387-398 (2007).

51. Fathi, A. and Naghsh-Nilchi, A.R. "Noise tolerant local binary pattern operator for efficient texture analysis", Pattern Recognit. Letters, 33(9), pp. 1093-1100 (2012).

52. Ren, J., Jiang, X. and Yuan, J. "Noise resistant local binary pattern with an embedded error correction mechanism", IEEE Trans. Image Process., 22(10), pp. 4049-4060 (2013).

53. Liu, L., Long, Y., Fieguth, P., Lao, S. and Zhao, G. "BRINT: Binary rotation invariant and noise tolerant texture classification", IEEE Trans. on Image Processing (2014).

54. Zhao, Y., Jia, W., XiangHuc, R. and Min, H. "Completed robust local binary pattern for texture classification", Neurocomputing, 106, pp. 68-76 (2013).

55. Kylberg G. and Sintorn I.M. "Evaluation of noise robustness for local binary pattern descriptors in texture classification", EURASIP Journal on Image and Video Processing, 17, pp. 1-20 (2013).

56. Zhang, Y., Wang, S., Ji, G. and Phillips, P. "Fruit classification using computer vision and feed forward neural network", Journal of Food Engineering, 143, pp. 167-177 (2014).

57. Unser, M. "Texture classification and segmentation using wavelet frames", IEEE Transaction on Image Processing, 4(11), pp. 1549-1560 (1995).

58. Guo, Z., Zhang, L. and Zhang, D. "A completed modeling of local binary pattern operator for texture classification", IEEE Trans. Image Process., 9(16), pp. 1657-1663 (2010).

59. Shakoor, M.H. and Tajeripour, F. "Circular mean filtering for textures noise reduction", Iranian Journal of Electrical \& Electronic Engineering, 11(3), pp. 195203 (2015).

60. Lazebnik, S., Schmid, C. and Ponce, J. "A sparse texture representation using local affine regions", IEEE Trans. Pattern Anal. Mach. Intell., 27(8), pp. 12651278 (2005).

61. Dana, K.J., Ginneken, B., Nayar, S.K. and Koenderink, J.J. "Reflectance and texture of real world surfaces", ACM Transactions on Graphics, 18(1), pp. 1-34 (1999).

62. Varma, M. and Zisserman, A. "A statistical approach to texture classification from single images", International Journal of Computer Vision, 62(1), pp. 61-81 (2005).

63. Varma, M. and Zisserman, A. "A statistical approach to material classification using image patch exemplars", IEEE Transactions on Pattern Analysis and Machine Intelligence, 31(11), pp. 2032-2047 (2009).

64. Lazebnik, S., Schmid, C. and Ponce, J. "A sparse texture representation using local affine regions", IEEE Trans. Pattern Anal. Mach. Intell., 27(8), pp. 12651278 (2005).

65. Makoto, N. and Takashi, M. "Edge preserving smoothing", Computer Graph Imag Proc, 9, pp. 394-407 (1979).

66. Zhang, Y. and Wu, L. "Improved image filter based on SPCNN", Science in China Series F-Information Sciences, 51(12), pp. 2115-2125 (2008).

67. Maani, R., Karla, S. and Yang, Y. "Noise robust rotation invariant features for texture classification", Pattern Recognition, 46, pp. 2103-2116 (2013).

68. Huang, D., Shan, C., Ardabilian, M., Wang, Y. and Chen, L. "Local binary patterns and its application to facial image analysis: A survey", IEEE Transactions on Systems Man, and Cybernetics, 4(5), pp. 765-781 (2011).

69. Shakoor, M.H. and Tajeripour, F. "Noise robust and rotation invariant entropy features for texture classification", Multimedia Tools and Applications, 75(6), pp. 1-36 (2016). DOI 10.1007/s11042-016-3455-6

\section{Biographies}

Mohammad Hossein Shakoor received the BS degree in Computer Engineering from Shiraz University, Shiraz, Iran, in 1998, and MS degree in Computer Architecture from Isfahan university, Isfahan, Iran, in 2003. Currently, he is pursuing PhD in Computer Engineering with the specialty of Artificial Intelligence at Shiraz University. His research interests include texture classification, pattern recognition, and computer vision.

Farshad Tajeripour received the BS and MS degrees in Electronic Engineering from Shiraz University, Shiraz, Iran, in 1994 and 1997, respectively. He received $\mathrm{PhD}$ degree in Electronic Engineering from Tarbiat Modarres University, Tehran, Iran, in 2009. Currently, he is an Assistant Professor at Shiraz University. His research interests include texture classification, pattern recognition, computer vision, and video processing. 